\title{
ON THE INTEGRAL REPRESENTATION OF RELAXED FUNCTIONALS WITH CONVEX BOUNDED CONSTRAINTS
}

\author{
OMAR ANZA HAFsA ${ }^{1}$
}

\begin{abstract}
We study the integral representation of relaxed functionals in the multi-dimensional calculus of variations, for integrands which are finite in a convex bounded set with nonempty interior and infinite elsewhere.
\end{abstract}

Mathematics Subject Classification. 49J45.

Received February 14, 2008.

Published online October 21, 2008.

\section{INTRODUCTION AND MAIN RESUltS}

Let $m, d \geq 1$ be two integers. Let $\Omega \subset \mathbb{R}^{d}$ be a nonempty open bounded domain with Lipschitz boundary. In this paper we consider the problem of the integral representation of

$$
\mathcal{I}(u)=\inf \left\{\liminf _{n \rightarrow+\infty} \int_{\Omega} f\left(\nabla u_{n}(x)\right) \mathrm{d} x: W^{1, \infty}\left(\Omega ; \mathbb{R}^{m}\right) \ni u_{n} \rightarrow u \text { in } L^{1}\right\},
$$

where $f: \mathbb{M}^{m \times d} \rightarrow[0,+\infty]$ is a Borel measurable function and with $\mathbb{M}^{m \times d}$ denotes the set of $m \times d$ matrices. We denote by $\operatorname{dom} f$ the effective domain of $f$, i.e., $\operatorname{dom} f=\left\{\xi \in \mathbb{M}^{m \times d}: f(\xi)<+\infty\right\}$. We are interested in integrands satisfying $\operatorname{dom} f \subset \bar{C}$, where $C$ is a convex bounded set with nonempty interior. The classical integral representation results of relaxed functionals in the vectorial case (i.e. when $\min \{d, m\} \geq 2$ ) require polynomial growth conditions (or at least integrands which are finite everywhere) on the integrands which do not allow us to deal with constraints on gradients. However, it is interesting for the applications in nonlinear elasticity to consider such constraints for problems, such as the elastic-plastic torsion problems and the modelling of rubber-like nonlinear elastomers as described by Carbone and De Arcangelis in [6]. In that book, we can find a detailed study of the problems of integral representation of relaxed functionals under constraints (not necessarily bounded) in the scalar case, i.e., $\min \{d, m\}=1$. In the vectorial case, and in the presence of some singular behaviors of the stored energy functions in nonlinear elasticity, we can find some relaxation results where the integrands can take the value $+\infty$, see $[2,3,5]$. Moreover, recently, in connection with relaxation problems in optimal control, Wagner [16] studies the relaxation of integral functional with the assumption that $f$ is continuous finite on $\bar{C}$, and infinite elsewhere.

\footnotetext{
Keywords and phrases. Relaxation, convex constraints, integral representation.

1 Université Montpellier II, UMR-CNRS 5508, LMGC, Place Eugène Bataillon, 34090 Montpellier, France.

Omar.Anza-Hafsa@univ-montp2.fr
} 
In this paper, we study the integral representation of $\mathcal{I}$ for two classes of integrands (we will make precise the assumptions later). Firstly, we consider a class of integrands which are locally bounded on int $C$ (the interior of $C$ ) and which allow us to consider singular behavior of the type

$$
f(\xi) \rightarrow+\infty \text { as } \xi \rightarrow \partial C \text { and } \operatorname{dom} f \subset \bar{C} .
$$

Secondly, we consider a class of integrands which are bounded on int $C$, which is in some sense a "complementary" class of the previous one. Similar to the classical relaxation results in the vectorial case, we will deal with the quasiconvex envelope of $f$. However, the definition of quasiconvex envelope is not obvious when $f$ is not everywhere finite. We avoid the difficulties connected with this problem by studying the possibility of monotone nondecreasing approximation of the quasiconvex envelope of $f$ by quasiconvex functions (which are finite by definition, see below).

\subsection{Some preliminary notions}

Following Morrey [12] we say that a function $g: \mathbb{M}^{m \times d} \rightarrow\left[0,+\infty\left[\right.\right.$ is quasiconvex at $\xi \in \mathbb{M}^{m \times d}$ if it is Borel measurable and

$$
g(\xi)=\inf \left\{\int_{Y} g(\xi+\nabla \phi(x)) \mathrm{d} x: \phi \in W_{0}^{1, \infty}\left(Y ; \mathbb{R}^{m}\right)\right\}
$$

where $Y=] 0,1\left[{ }^{d}\right.$ is the unit cube in $\mathbb{R}^{d}$. If $g$ is quasiconvex at every $\xi \in \mathbb{M}^{m \times d}$ then $g$ is said quasiconvex. If $g$ is quasiconvex then it is continuous (see for instance Dacorogna [7]).

Let us define by $\mathcal{Q} f: \mathbb{M}^{m \times d} \rightarrow[0,+\infty]$ the quasiconvex envelope of $f$ defined by

$$
\mathcal{Q} f(\xi)=\sup \left\{g(\xi): g: \mathbb{M}^{m \times d} \rightarrow[0,+\infty[\text { is quasiconvex and } g \leq f\} .\right.
$$

Note that $\mathcal{Q} f$ is lower semicontinuous as pointwise supremum of continuous functions, and satisfies

$$
\mathcal{Q} f(\xi)=\inf \left\{\int_{Y} \mathcal{Q} f(\xi+\nabla \phi(x)) \mathrm{d} x: \phi \in W_{0}^{1, \infty}\left(Y ; \mathbb{R}^{m}\right)\right\}
$$

for all $\xi \in \mathbb{M}^{m \times d}$.

Let $h: \mathbb{M}^{m \times d} \rightarrow[0,+\infty]$ be a Borel measurable function. We say that $h$ is $p$-sup-quasiconvex if there exist $p \in\left[1,+\infty\left[\right.\right.$ and a nondecreasing sequence $\left\{h_{n}\right\}_{n \in \mathbb{N}}, h_{n}: \mathbb{M}^{m \times d} \rightarrow[0,+\infty[$ such that

(i) $h_{n}$ is quasiconvex for all $n \in \mathbb{N}$;

(ii) for every $n \in \mathbb{N}$ there exists $\alpha_{n}>0$ such that $h_{n}(\xi) \leq \alpha_{n}\left(1+|\xi|^{p}\right)$ for all $\xi \in \mathbb{M}^{m \times d}$;

(iii) for every $\xi \in \mathbb{M}^{m \times d}$ we have that

$$
\sup \left\{h_{n}(\xi): n \in \mathbb{N}\right\}=h(\xi) .
$$

It is easy to see that if $h$ is $p$-sup-quasiconvex then it is lower semicontinuous as pointwise supremum of continuous functions and satisfies

$$
h(\xi)=\inf \left\{\int_{Y} h(\xi+\nabla \phi(x)) \mathrm{d} x: \phi \in W_{0}^{1, \infty}\left(Y ; \mathbb{R}^{m}\right)\right\}
$$

for all $\xi \in \mathbb{M}^{m \times d}$.

Define $\mathcal{Z} f: \mathbb{M}^{m \times d} \rightarrow[0,+\infty]$ by

$$
\mathcal{Z} f(\xi)=\inf \left\{\int_{Y} f(\xi+\nabla \phi(x)) \mathrm{d} x: \phi \in W_{0}^{1, \infty}\left(Y ; \mathbb{R}^{m}\right)\right\} .
$$


In fact $\mathcal{Z} f$ does not depend on $Y$, see Lemma A.1. If $\mathcal{Z} f$ is everywhere finite, then $\mathcal{Z} f=\mathcal{Q} f$ (see Lem. A.2), and $\mathcal{Z} f$ is called the Dacorogna formula of the quasiconvex envelope of $f$.

In the rest of this paper we will use frequently some properties of convex sets which are summarized as line segment principle by Rockafellar and Wets [14], Theorem 2.33.

Line segment principle (l.s.p.):

Let $C \subset \mathbb{M}^{m \times d}$ be a bounded convex set with $0 \in \operatorname{int} C$. Then

$$
\operatorname{int} \bar{C}=\operatorname{int} C, \quad \overline{\operatorname{int} C}=\bar{C}, \text { and } t \bar{C} \subset \operatorname{int} C \text { for all } t \in[0,1[.
$$

\subsection{Main results}

Let $C \subset \mathbb{M}^{m \times d}$ be a bounded convex set with nonempty interior. To simplify the statements we will assume through the paper that $0 \in \operatorname{int} C$. Let $f: \mathbb{M}^{m \times d} \rightarrow[0,+\infty]$ be a Borel measurable function such that $\operatorname{dom} f \subset \bar{C}$. We consider the following assertion:

$\left(\mathscr{H}_{0}\right)$ for every $\varepsilon>0$ there exists $\eta>0$ such that for every $\xi \in \operatorname{int} C$ and every $t \in[0,1[$ we have

$$
1-t \leq \eta \Longrightarrow \mathcal{Z} f(t \xi) \leq \mathcal{Z} f(\xi)+\varepsilon .
$$

1.2.1. Integral representation for integrands locally bounded on $\operatorname{int} C$

Consider the following assertions:

$\left(\mathscr{H}_{1}\right) f$ is locally bounded on $\operatorname{int} C$, i.e., $\sup \{f(\xi): \xi \in K\}<+\infty$ for all compact sets $K \subset \operatorname{int} C$;

$\left(\mathscr{H}_{2}\right)$ for every $a>0$ there exists a compact set $K_{a} \subset \operatorname{int} C$ such that for every $\xi \in \bar{C}$,

$$
\xi \notin K_{a} \Longrightarrow \mathcal{Z} f(\xi) \geq a .
$$

Theorem 1.1. Assume that $\left(\mathscr{H}_{0}\right),\left(\mathscr{H}_{1}\right)$ and $\left(\mathscr{H}_{2}\right)$ hold. Then $\mathcal{Q} f$ is 1-sup-quasiconvex and we have the representation formula

$$
\mathcal{Q} f(\xi)= \begin{cases}\mathcal{Z} f(\xi) & \text { if } \xi \in \operatorname{int} C \\ +\infty & \text { otherwise. }\end{cases}
$$

Theorem 1.2. Assume that $\left(\mathscr{H}_{0}\right),\left(\mathscr{H}_{1}\right)$ and $\left(\mathscr{H}_{2}\right)$ hold. Then

$$
\mathcal{I}(u)=\left\{\begin{array}{cl}
\int_{\Omega} \mathcal{Q} f(\nabla u(x)) \mathrm{d} x & \text { if } u \in W^{1, \infty}\left(\Omega ; \mathbb{R}^{m}\right) \\
+\infty & \text { if } u \in L^{1}\left(\Omega ; \mathbb{R}^{m}\right) \backslash W^{1, \infty}\left(\Omega ; \mathbb{R}^{m}\right) .
\end{array}\right.
$$

\subsubsection{Integral representation for integrands bounded on $\operatorname{int} C$}

Consider the following assertions:

$\left(\mathscr{H}_{3}\right) f$ is bounded on $\operatorname{int} C$, i.e., $\sup \{f(\xi): \xi \in \operatorname{int} C\}<+\infty$;

$\left(\mathscr{H}_{4}\right)$ for every $\xi \in \partial C$ we have $\lim \inf _{[0,1[\ni t \rightarrow 1} f(t \xi) \leq f(\xi)$.

Theorem 1.3. Assume that $\left(\mathscr{H}_{0}\right),\left(\mathscr{H}_{3}\right)$ and $\left(\mathscr{H}_{4}\right)$ hold. Then $\mathcal{Q f}$ is 1-sup-quasiconvex and we have the representation formula

$$
\mathcal{Q} f(\xi)=\overline{\mathcal{Z} f}(\xi)=\left\{\begin{array}{cl}
\mathcal{Z} f(\xi) & \text { if } \xi \in \operatorname{int} C \\
\lim _{[0,1[\ni t \rightarrow 1} \mathcal{Z} f(t \xi) & \text { if } \xi \in \partial C \\
+\infty & \text { otherwise. }
\end{array}\right.
$$

The above representation formula for $\mathcal{Q} f$ was found by Wagner in $[15](\overline{\mathcal{Z} f}$ denotes the lower semicontinuous envelope of $\mathcal{Z} f$ ). 
Theorem 1.4. Assume that $\left(\mathscr{H}_{0}\right),\left(\mathscr{H}_{3}\right)$ and $\left(\mathscr{H}_{4}\right)$ hold. Then

$$
\mathcal{I}(u)=\left\{\begin{array}{cl}
\int_{\Omega} \mathcal{Q} f(\nabla u(x)) \mathrm{d} x & \text { if } u \in W^{1, \infty}\left(\Omega ; \mathbb{R}^{m}\right) \\
+\infty & \text { if } u \in L^{1}\left(\Omega ; \mathbb{R}^{m}\right) \backslash W^{1, \infty}\left(\Omega ; \mathbb{R}^{m}\right) .
\end{array}\right.
$$

Remark 1.1. (i) In [6], Theorem 10.2.4, Carbone and De Arcangelis use similar assumption $\left(\mathscr{H}_{0}\right)$ for the problem of the integral representation of relaxed functionals, in the scalar case. They translate that assumption as a type of "uniform radial upper semicontinuity" on int $C$ (see Rem. 10.1.1 in [6]).

The assertion $\left(\mathscr{H}_{0}\right)$ is satisfied when for instance $\operatorname{int} C \subset \operatorname{dom} f \subset \bar{C}$, and for every $\varepsilon>0$ there exists $\eta>0$ such that for every $\xi \in \operatorname{dom} f$ and every $t \in[0,1[$ it holds

$$
1-t \leq \eta \Longrightarrow f(t \xi) \leq f(\xi)+\frac{\varepsilon}{2}
$$

Indeed, take $\varepsilon>0, \eta>0$ as above. Let $t \in[0,1[$ be such that $1-t \leq \eta$. Let $\xi \in \operatorname{int} C$. Then we have that $\mathcal{Z} f(\xi) \leq f(\xi)<+\infty$ and there exists $\phi_{\varepsilon} \in W_{0}^{1, \infty}\left(Y ; \mathbb{R}^{m}\right)$ such that

$$
\frac{\varepsilon}{2}+\mathcal{Z} f(\xi) \geq \int_{Y} f\left(\xi+\nabla \phi_{\varepsilon}(x)\right) \mathrm{d} x
$$

Thus $\xi+\nabla \phi_{\varepsilon}(x) \in \operatorname{dom} f$ a.e. in $Y$, and we have

$$
\mathcal{Z} f(t \xi) \leq \int_{Y} f\left(t\left(\xi+\nabla \phi_{\varepsilon}(x)\right)\right) \mathrm{d} x \leq \frac{\varepsilon}{2}+\int_{Y} f\left(\xi+\nabla \phi_{\varepsilon}(x)\right) \mathrm{d} x .
$$

By (1.3), we obtain $\mathcal{Z} f(t \xi) \leq \mathcal{Z} f(\xi)+\varepsilon$.

(ii) The assertion $\left(\mathscr{H}_{2}\right)$ is satisfied when for instance $f \geq \psi$ for some convex function $\psi$ satisfying for every $a>0$ there exists a compact set $K_{a} \subset \operatorname{int} C$ such that for every $\xi \in \bar{C}$,

$$
\xi \notin K_{a} \Longrightarrow \psi(\xi) \geq a
$$

(iii) Let $f: \mathbb{M}^{m \times d} \rightarrow[0,+\infty]$ be defined by

$$
f(\xi)= \begin{cases}g(\xi)+\frac{1}{1-|\xi|} & \text { if }|\xi|<1 \\ +\infty & \text { otherwise }\end{cases}
$$

where $g: \mathbb{M}^{m \times d} \rightarrow\left[0,+\infty\left[\right.\right.$ is uniformly continuous. In view of (i) and (ii), we have that $\left(\mathscr{H}_{0}\right),\left(\mathscr{H}_{1}\right)$ and $\left(\mathscr{H}_{2}\right)$ are satisfied.

(iv) Note that $\left(\mathscr{H}_{3}\right)$ and $\left(\mathscr{H}_{4}\right)$ are satisfied if, for instance, $f\left\lfloor_{\bar{C}} \in \mathcal{C}(\bar{C})\right.$.

\subsection{Comments on $\mathcal{Z} f$}

To our best knowledge, the formula $\mathcal{Z} f$ first appeared for arbitrary Borel measurable function $f$ in Ball and Murat [4], p. 240. Then Fonseca in [10] studied the rank-one convexity property of $\mathcal{Z} f$ for arbitrary Borel measurable $f$, in particular she showed that $\mathcal{Z} f$ is continuous on $\operatorname{int}(\operatorname{dom} f)$ (Thm. 2.17 and Prop. 2.3). Later Kinderlehrer and Pedregal in [11] considered $\mathcal{Z} f$ for functions $f$ satisfying

$$
f\left\lfloor_ { \overline { B } } \in \mathcal { C } ( \overline { B } ) \text { and } f \left\lfloor_{\mathbb{M}^{m \times d} \backslash \bar{B}}=+\infty\right.\right.
$$


with $B=\left\{\xi \in \mathbb{M}^{m \times d}:|\xi|<1\right\} \subset \mathbb{M}^{m \times d}$ is the unit ball. They showed, in particular, that $\mathcal{Z} f$ satisfies (1.4) and $\mathcal{Z}(\mathcal{Z} f)=\mathcal{Z} f$ in their Proposition 7.2. In the paper [8] Dacorogna and Marcellini studied $\mathcal{Z} f$ in Theorem 7.2 for the class of functions satisfying (1.4) where $B$ is replaced by an arbitrary compact convex set $K \subset \mathbb{M}^{m \times d}$ with nonempty interior. Recently, the work of Wagner in [15] gives a detailed study of $\mathcal{Z} f$ with $f$ satisfying the same assumptions as in Dacorogna and Marcellini [8].

\subsection{Outline}

An outline of the paper is shown as follows: We start by some preliminary lemmas, where we are mainly concerned with establishing some properties of $\mathcal{Z} f$. The proofs of Theorems 1.1 and 1.3 are achieved by using some arguments of Müller [13]. We give the proofs of Theorems 1.2 and 1.4 by dividing them into two steps. The proof of the lower bound follows easily after we have shown that $\mathcal{Q} f$ is 1 -sup-quasiconvex. To prove the upper bound, we use an approximation result due to Dacorogna and Marcellini [8]. In the appendix, we give some results concerning $\mathcal{Z} f$.

\section{Preliminaries}

\section{Lemma 2.1.}

(i) For every increasing sequence $\left\{t_{n}\right\}_{n \in \mathbb{N}^{*}} \subset\left[0,1\right.$ [ satisfying $\lim _{n \rightarrow+\infty} t_{n}=1$, it holds

$$
\operatorname{int} C=\bigcup_{t \in[0,1[} t \bar{C}=\bigcup_{n \in \mathbb{N}^{\star}} t_{n} \bar{C} \text {, and } \operatorname{int} C=\bigcup_{t \in[0,1[} t \operatorname{int} C=\bigcup_{n \in \mathbb{N}^{\star}} t_{n} \operatorname{int} C .
$$

(ii) If $K \subset \operatorname{int} C$ is compact then $K \subset t$ int $C$ for some $t \in[0,1[$.

(iii) The function $f$ is locally bounded on $\operatorname{int} C$ if and only if $\sup \{f(\xi): \xi \in t \bar{C}\}<+\infty$ for all $t \in[0,1[$.

(iv) Assume that $f$ is locally bounded on $\operatorname{int} C$. Then $\left(\mathscr{H}_{2}\right)$ holds if and only if there exists an increasing sequence such that $\left[0,1\left[\ni t_{n} \rightarrow 1\right.\right.$, and for every $n \geq 1$

$$
\inf _{\xi \in \bar{C} \backslash t_{n} \bar{C}} \mathcal{Z} f(\xi) \geq n
$$

Proof. (i) Let $\left\{t_{n}\right\}_{n \in \mathbb{N}^{*}} \subset\left[0,1\left[\right.\right.$ be an increasing sequence such that $\lim _{n \rightarrow+\infty} t_{n}=1$. Let $\xi \in \operatorname{int} C$ and set $D=\cup_{n \geq 1} t_{n} \bar{C}$, then $t_{n} \xi \in D$ for all $n \in \mathbb{N}^{*}$ by l.s.p. We deduce that $\xi \in \bar{D}$ and it holds

$$
\operatorname{int} D \subset D \subset \operatorname{int} C \subset \bar{D}
$$

Note that firstly $D$ is convex since $\left\{t_{n}\right\}_{n \in \mathbb{N}^{*}}$ is increasing, and secondly $0 \in \operatorname{int} D$. Thus, by $\operatorname{l.s.p.}$, int $D=\operatorname{int} \bar{D}$, and by (2.1) it follows that $\operatorname{int} C=\operatorname{int} D=D$ and we obtain

$$
\operatorname{int} C=\bigcup_{t \in[0,1[} t \bar{C}=\bigcup_{n \in \mathbb{N}^{\star}} t_{n} \bar{C}
$$

Now, note that by l.s.p., it holds $t_{n} \bar{C} \subset t_{n+1} \operatorname{int} C$ for all $n \in \mathbb{N}^{*}$. Thus for every $n \in \mathbb{N}^{*}$ we have $t_{n} \bar{C} \subset$ $\cup_{n \in \mathbb{N}^{*}} t_{n} \operatorname{int} C$, hence $\operatorname{int} C \subset \cup_{n \in \mathbb{N}^{*}} t_{n} \operatorname{int} C$.

(ii) Let $K \subset \operatorname{int} C$ be a compact set. Assume that for every $n \in \mathbb{N}^{*}$ there exists $x_{n} \in K$ and $x_{n} \notin t_{n}$ int $C$ where $t_{n}=\frac{n-1}{n}$. By compactness, there exists a converging subsequence $K \ni x_{\sigma(n)} \rightarrow x \in K$ as $n \rightarrow+\infty$. By (i) we have $t_{\sigma(1)} \operatorname{int} C \subset \cdots \subset t_{\sigma(n)} \operatorname{int} C \subset \cdots \subset \cup_{n \geq 1} t_{\sigma(n)} \operatorname{int} C=\operatorname{int} C$. For every $k, n \in \mathbb{N}^{*}$

$$
x_{\sigma(n+k)} \in K \backslash t_{\sigma(n+k)} \operatorname{int} C \subset K \backslash t_{\sigma(n)} \operatorname{int} C .
$$

Letting $k \rightarrow+\infty$, we obtain $x \in K \backslash \cup_{n \in \mathbb{N}^{*}} t_{\sigma(n)}$ int $C=\emptyset$, which is impossible since $K$ is compact. 
(iii) Follows from (ii).

(iv) By $\left(\mathscr{H}_{2}\right)$, we can find a sequence of compact set $\left\{K_{n}\right\}_{n \in \mathbb{N}^{*}} \subset \operatorname{int} C$ such that

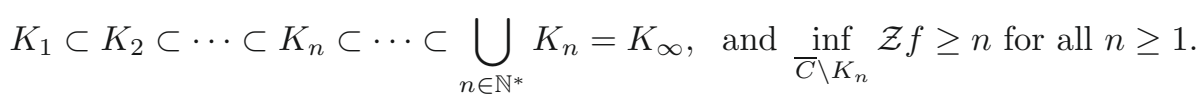

Thus $\inf _{\bar{C} \backslash K_{\infty}} \mathcal{Z} f=+\infty$. Assume that $K_{\infty} \neq \operatorname{int} C$, then there exists $\xi_{0} \in \operatorname{int} C \backslash K_{\infty}$ such that $\mathcal{Z} f\left(\xi_{0}\right)=+\infty$. But by (i) and (iii) we obtain $+\infty=\mathcal{Z} f\left(\xi_{0}\right) \leq f\left(\xi_{0}\right)<+\infty$ which is impossible. Thus $K_{\infty}=\operatorname{int} C$. By (ii), we can build an increasing sequence $\left\{t_{n}\right\}_{n \in \mathbb{N}^{*}} \subset\left[0,1\left[\right.\right.$ such that $K_{n} \subset t_{n} \bar{C}$ for all $n \in \mathbb{N}^{*}$. It follows that $\operatorname{int} C=K_{\infty}=\cup_{n \geq 1} t_{n} \bar{C}$ and therefore the sequence $t_{n} \rightarrow 1$ as $n \rightarrow+\infty$, indeed we cannot have $\tau=\sup _{n \geq 1} t_{n}=$ $\lim _{n \rightarrow+\infty} t_{n}<1$, otherwise, by l.s.p. $\operatorname{int} C \subset \tau \operatorname{int} C$ which is impossible $\operatorname{since} \operatorname{int} C \neq \emptyset$. We also have for every $n \in \mathbb{N}^{*}$

$$
\inf _{\xi \in \bar{C} \backslash t_{n} \bar{C}} \mathcal{Z} f(\xi) \geq \inf _{\xi \in \bar{C} \backslash K_{n}} \mathcal{Z} f(\xi) \geq n
$$

The other implication is easier. Let $a>0$. Let $n \geq a$ and choose $K_{a}=t_{n} \bar{C}$ then $\inf _{\bar{C} \backslash K_{a}} \mathcal{Z} f \geq n \geq a$. The proof is complete.

Lemma 2.2. Assume that $\left(\mathscr{H}_{1}\right)$ holds. Then

$$
\operatorname{int} C \subset \operatorname{dom} f \subset \operatorname{dom} \mathcal{Z} f \subset \operatorname{dom} \overline{\mathcal{Z} f} \subset \operatorname{dom} \mathcal{Q} f \subset \bar{C}
$$

and

$$
\operatorname{int}(\operatorname{dom} f)=\operatorname{int}(\operatorname{dom} \mathcal{Z} f)=\operatorname{int}(\operatorname{dom} \overline{\mathcal{Z} f})=\operatorname{int}(\operatorname{dom} \mathcal{Q} f)=\operatorname{int} C .
$$

Proof. By definition of $\mathcal{Q} f$ and $\mathcal{Z} f$ we have

$$
\mathcal{Q} f \leq \overline{\mathcal{Z} f} \leq \mathcal{Z} f \leq f
$$

By Lemma 2.1 (i) and (ii), if $\xi \in \operatorname{int} C$ then there exists $t \in[0,1[$ such that $\xi \in t \bar{C}$ and

$$
\mathcal{Q} f(\xi) \leq \overline{\mathcal{Z} f}(\xi) \leq \mathcal{Z} f(\xi) \leq f(\xi) \leq \sup _{\zeta \in t \bar{C}} f(\zeta)<+\infty
$$

We deduce $\operatorname{int} C \subset \operatorname{dom} f \subset \operatorname{dom} \mathcal{Z} f \subset \operatorname{dom} \overline{\mathcal{Z} f} \subset \operatorname{dom} \mathcal{Q} f$. Now, we will show dom $\mathcal{Q} f \subset \bar{C}$. For each $n \in \mathbb{N}$, consider the function $g_{n}: \mathbb{M}^{m \times d} \rightarrow\left[0,+\infty\right.$ [ defined by $g_{n}(\xi)=n \operatorname{dist}(\xi, \bar{C})$. It is easy to see that $g_{n}$ is convex and then quasiconvex since Jensen inequality, and $g_{n} \leq f$ for all $n \in \mathbb{N}$. Thus $g_{n} \leq \mathcal{Q} f$ for all $n \in \mathbb{N}$, and the inclusion $\operatorname{dom} \mathcal{Q} f \subset \bar{C}$ follows by noticing that

$$
\sup _{n \in \mathbb{N}} g_{n}(\xi)= \begin{cases}0 & \text { if } \xi \in \bar{C} \\ +\infty & \text { otherwise. }\end{cases}
$$

The second sequence of equalities follows by applying l.s.p. The proof is finished.

Lemma 2.3. Assume that $\left(\mathscr{H}_{1}\right)$ holds. Then $\mathcal{Z} f$ is continuous in int $C$.

Proof. By Proposition 2.3 and Theorem 2.17 in [10], we deduce that $\mathcal{Z} f$ is continuous on every open set $U \subset \operatorname{dom} f$. In particular, $\mathcal{Z} f$ is continuous on $\operatorname{int}(\operatorname{dom} f)$ which coincide with int $C$ since Lemma 2.2.

The following lemma is essentially due to Wagner [15]. We prove it by borrowing some arguments of the proof of Theorem 3.12. 2) in [15].

Lemma 2.4. Assume that $\left(\mathscr{H}_{0}\right)$ and $\left(\mathscr{H}_{1}\right)$ hold. Then $\lim _{[0,1[\ni t \rightarrow 1} \mathcal{Z} f(t \xi) \in[0,+\infty]$ for all $\xi \in \partial C$. 
Proof. Let $\xi \in \partial C$. Set $\lambda=\lim \sup _{[0,1[\ni t \rightarrow 1} \mathcal{Z} f(t \xi)$ and $\mu=\liminf _{[0,1[\ni t \rightarrow 1} \mathcal{Z} f(t \xi)$. If $\mu=+\infty$ then

$$
\lambda=\mu=\lim _{[0,1[\ni t \rightarrow 1} \mathcal{Z} f(t \xi)=+\infty .
$$

Assume that $\mu<+\infty$. We have two possibilities, either $\lambda=+\infty$ or $\lambda<+\infty$.

Suppose that $\lambda=+\infty$. Consider two sequences $\left\{t_{n}\right\}_{n \in \mathbb{N}^{*}},\left\{\tau_{n}\right\}_{n \in \mathbb{N}^{*}} \subset\left[0,1\left[\right.\right.$ such that $t_{n} \rightarrow 1$ and $\tau_{n} \rightarrow 1$ as $n \rightarrow+\infty$ satisfying

$$
\lambda=\lim _{n \rightarrow+\infty} \mathcal{Z} f\left(t_{n} \xi\right) \text { and } \mu=\lim _{n \rightarrow+\infty} \mathcal{Z} f\left(\tau_{n} \xi\right) .
$$

We can find two increasing functions $\sigma, \sigma^{\prime}: \mathbb{N}^{*} \rightarrow \mathbb{N}^{*}$ such that for every $n \in \mathbb{N}^{*}$

$$
1-\frac{1}{n} \leq t_{\sigma(n)}<\tau_{\sigma^{\prime}(n)}<1 .
$$

Let $\varepsilon>0$. There exists $N_{0} \in \mathbb{N}^{*}$ such that for every $n \geq N_{0}$ it holds

$$
\mathcal{Z} f\left(t_{\sigma(n)} \xi\right) \geq 1+\varepsilon+\mu \text { and }\left|\mathcal{Z} f\left(\tau_{\sigma^{\prime}(n)} \xi\right)-\mu\right| \leq \frac{\varepsilon}{2} .
$$

By $\left(\mathscr{H}_{0}\right)$ there exists $\eta>0$ such that for every $\xi \in \operatorname{int} C$ and every $t \in[0,1[$ it holds

$$
1-t \leq \eta \Longrightarrow \mathcal{Z} f(t \xi) \leq \mathcal{Z} f(\xi)+\frac{\varepsilon}{2} \text {. }
$$

Choose an integer $n \geq \max \left\{2, N_{0}, \eta^{-1}\right\}$. Then it holds that

$$
\tau_{\sigma^{\prime}(n)}>0 \text { and } 1-\frac{t_{\sigma(n)}}{\tau_{\sigma^{\prime}(n)}} \leq \eta .
$$

Therefore, by (2.2), (2.4), (2.3) and l.s.p. we obtain

$$
\begin{aligned}
1+\varepsilon+\mu & \leq \mathcal{Z} f\left(t_{\sigma(n)} \xi\right)-\mathcal{Z} f\left(\tau_{\sigma^{\prime}(n)} \xi\right)+\mathcal{Z} f\left(\tau_{\sigma^{\prime}(n)} \xi\right) \\
& =\mathcal{Z} f\left(\frac{t_{\sigma(n)}}{\tau_{\sigma^{\prime}(n)}} \tau_{\sigma^{\prime}(n)} \xi\right)-\mathcal{Z} f\left(\tau_{\sigma^{\prime}(n)} \xi\right)+\mathcal{Z} f\left(\tau_{\sigma^{\prime}(n)} \xi\right) \\
& \leq \varepsilon+\mu,
\end{aligned}
$$

which is impossible. It means that if $\mu<+\infty$ then $\lambda<+\infty$.

Now, we will show that in this case $\mu=\lambda$. Consider $\left\{t_{n}\right\}_{n \in \mathbb{N}^{*}},\left\{\tau_{n}\right\}_{n \in \mathbb{N}^{*}} \subset\left[0,1\left[\right.\right.$ such that $t_{n} \rightarrow 1$ and $\tau_{n} \rightarrow 1$ as $n \rightarrow+\infty$ satisfying

$$
\lambda=\lim _{n \rightarrow+\infty} \mathcal{Z} f\left(t_{n} \xi\right) \text { and } \mu=\lim _{n \rightarrow+\infty} \mathcal{Z} f\left(\tau_{n} \xi\right) .
$$

As above we can find two subsequences such that for every $n \in \mathbb{N}^{*}$

$$
1-\frac{1}{n} \leq t_{\sigma(n)}<\tau_{\sigma^{\prime}(n)}<1 .
$$

Let $\varepsilon>0$. There exists $N_{0} \in \mathbb{N}^{*}$ such that for every $n \geq N_{0}$ it holds

$$
\left|\mathcal{Z} f\left(t_{\sigma(n)} \xi\right)-\lambda\right| \leq \frac{\varepsilon}{3} \text { and }\left|\mathcal{Z} f\left(\tau_{\sigma^{\prime}(n)} \xi\right)-\mu\right| \leq \frac{\varepsilon}{3} .
$$

By $\left(\mathscr{H}_{0}\right)$ there exists $\eta>0$ such that for every $\xi \in \operatorname{int} C$ and every $t \in[0,1[$ it holds

$$
1-t \leq \eta \Longrightarrow \mathcal{Z} f(t \xi) \leq \mathcal{Z} f(\xi)+\frac{\varepsilon}{3} \text {. }
$$


Choose an integer $n \geq \max \left\{2, N_{0}, \eta^{-1}\right\}$. Then it holds that

$$
\tau_{\sigma^{\prime}(n)}>0 \text { and } 1-\frac{t_{\sigma(n)}}{\tau_{\sigma^{\prime}(n)}} \leq \eta
$$

Therefore, by (2.5), (2.7), (2.6) and l.s.p. we obtain

$$
\begin{aligned}
0 \leq \lambda-\mu & =\lambda-\mathcal{Z} f\left(t_{\sigma(n)} \xi\right)+\mathcal{Z} f\left(t_{\sigma(n)} \xi\right)-\mathcal{Z} f\left(\tau_{\sigma^{\prime}(n)} \xi\right)+\mathcal{Z} f\left(\tau_{\sigma^{\prime}(n)} \xi\right)-\mu \\
& \leq \frac{2 \varepsilon}{3}+\mathcal{Z} f\left(\frac{t_{\sigma(n)}}{\tau_{\sigma^{\prime}(n)}} \tau_{\sigma^{\prime}(n)} \xi\right)-\mathcal{Z} f\left(\tau_{\sigma^{\prime}(n)} \xi\right) \leq \varepsilon
\end{aligned}
$$

The proof is complete since $\varepsilon>0$ is arbitrary.

$\operatorname{Under}\left(\mathscr{H}_{0}\right)$ and $\left(\mathscr{H}_{1}\right)$ we will denote by $\widehat{\mathcal{Z} f}: \mathbb{M}^{m \times d} \rightarrow[0,+\infty]$ the function defined by

$$
\widehat{\mathcal{Z f}}(\xi)= \begin{cases}\mathcal{Z} f(\xi) & \text { if } \xi \in \operatorname{int} C \\ \lim _{[0,1[\ni t \rightarrow 1} \mathcal{Z} f(t \xi) & \text { if } \xi \in \partial C \\ +\infty & \text { otherwise }\end{cases}
$$

Lemma 2.5. Assume that $\left(\mathscr{H}_{0}\right)$ and $\left(\mathscr{H}_{1}\right)$ hold. Then for every $\varepsilon>0$ there exists $\eta>0$ such that for every $\xi \in \bar{C}$ and $t \in[0,1[$ it holds

$$
1-t \leq \eta \Longrightarrow \mathcal{Z} f(t \xi) \leq \widehat{\mathcal{Z} f}(\xi)+\varepsilon
$$

Proof. Let $\left\{\tau_{n}\right\}_{n \in \mathbb{N}} \subset\left[0,1\left[\right.\right.$ be such that $\tau_{n} \rightarrow 1$ as $n \rightarrow+\infty$. Let $\varepsilon>0$. By $\left(\mathscr{H}_{0}\right)$ and l.s.p., there exists $\eta>0$ such that for every $n \in \mathbb{N}$, every $t \in[0,1[$ and $\xi \in \bar{C}$ it holds

$$
1-t \leq \eta \Longrightarrow \mathcal{Z} f\left(t \tau_{n} \xi\right) \leq \mathcal{Z} f\left(\tau_{n} \xi\right)+\varepsilon
$$

Since $\mathcal{Z} f$ is continuous in $\operatorname{int} C$ by Lemma 2.3 and using Lemma 2.4, we deduce for every $t \in[0,1[$ and $\xi \in \bar{C}$

$$
1-t \leq \eta \Longrightarrow \lim _{n \rightarrow+\infty} \mathcal{Z} f\left(t \tau_{n} \xi\right)=\mathcal{Z} f(t \xi) \leq \lim _{n \rightarrow+\infty} \mathcal{Z} f\left(\tau_{n} \xi\right)+\varepsilon=\widehat{\mathcal{Z} f}(\xi)+\varepsilon
$$

The proof is complete.

Lemma 2.6. Assume that $\left(\mathscr{H}_{0}\right)$ and $\left(\mathscr{H}_{1}\right)$ hold. Then for every $\xi \in \mathbb{M}^{m \times d}$

$$
\widehat{\mathcal{Z} f}(\xi)=\inf \left\{\int_{Y} \widehat{\mathcal{Z} f}(\xi+\nabla \phi(x)) \mathrm{d} x: \phi \in W_{0}^{1, \infty}\left(Y ; \mathbb{R}^{m}\right)\right\} .
$$

Proof. It is enough to prove

$$
\widehat{\mathcal{Z} f}(\xi) \leq \inf \left\{\int_{Y} \widehat{\mathcal{Z} f}(\xi+\nabla \phi(x)) \mathrm{d} x: \phi \in W_{0}^{1, \infty}\left(Y ; \mathbb{R}^{m}\right)\right\} .
$$

Let $\xi \in \mathbb{M}^{m \times d}$ and $\phi \in W_{0}^{1, \infty}\left(Y ; \mathbb{R}^{m}\right)$ such that

$$
\int_{Y} \widehat{\mathcal{Z} f}(\xi+\nabla \phi(x)) \mathrm{d} x<+\infty
$$


Then $\xi+\nabla \phi(x) \in \bar{C}$ a.e. in $Y$, and so $\xi \in \bar{C}$ since $\bar{C}$ is convex. Let $n \geq 1$ and $t_{n}=1-\frac{1}{n}$. Let $Y \ni x \mapsto$ $t_{n} \xi x+t_{n} \phi(x) \in u_{t_{n} \xi}+W_{0}^{1, \infty}\left(Y ; \mathbb{R}^{m}\right)$, where $u_{t_{n} \xi}(x)=t_{n} \xi x$ for $x \in Y$. By l.s.p., it holds that $\nabla u_{n}(x) \in t_{n} \bar{C}$ a.e. in $Y$. Let $\varepsilon>0$. By Lemma 2.5, there exists $\eta>0$ such that for every $\xi \in \bar{C}$ and $t \in[0,1[$, if $1-t<\eta$ then $\mathcal{Z} f(t \xi) \leq \widehat{\mathcal{Z} f}(\xi)+\varepsilon$. Choose $n(\varepsilon) \in \mathbb{N}^{*}$ such that $1-n^{-1}<\eta$ for all $n \geq n(\varepsilon)$. Thus, for every $n \geq n(\varepsilon)$

$$
\int_{Y} \mathcal{Z} f\left(t_{n} \xi+t_{n} \nabla \phi(x)\right) \mathrm{d} x \leq \varepsilon+\int_{Y} \widehat{\mathcal{Z} f}(\xi+\nabla \phi(x)) \mathrm{d} x .
$$

Applying Lemma 6.1, for every $n \geq n(\varepsilon)$, we can find $\left\{v_{k}^{n}\right\}_{k \geq 1} \subset u_{t_{n} \xi}+W_{0}^{1, \infty}\left(Y ; \mathbb{R}^{m}\right)$ such that

$$
\limsup _{k \rightarrow+\infty} \int_{Y} f\left(\nabla v_{k}^{n}(x)-t_{n} \xi+t_{n} \xi\right) \mathrm{d} x \leq \int_{Y} \mathcal{Z} f\left(t_{n} \xi+t_{n} \nabla \phi(x)\right) \mathrm{d} x .
$$

Thus, since $\mathcal{Z} f \leq f$ and $v_{k}^{n}-u_{t_{n} \xi} \in W_{0}^{1, \infty}\left(Y ; \mathbb{R}^{m}\right)$ we obtain

$$
\mathcal{Z} f\left(t_{n} \xi\right) \leq \varepsilon+\int_{\Omega} \widehat{\mathcal{Z} f}(\xi+\nabla \phi(x)) \mathrm{d} x .
$$

Letting $n \rightarrow+\infty$ and using Lemmas 2.3 and 2.4, we have

$$
\widehat{\mathcal{Z} f}(\xi) \leq \varepsilon+\int_{\Omega} \widehat{\mathcal{Z} f}(\xi+\nabla \phi(x)) \mathrm{d} x
$$

We obtain the desired result, since $\varepsilon>0$ is arbitrary.

Lemma 2.7 (Müller [13], Thm. 4). Let $K \subset \mathbb{M}^{m \times d}$ be a compact convex set. Let $\Omega \subset \mathbb{R}^{d}$ be a bounded, open set with Lipschitz boundary. Let $\left\{u_{n}\right\}_{n \in \mathbb{N}} \subset W^{1, \infty}\left(\Omega ; \mathbb{R}^{m}\right)$ be a sequence satisfying

$$
u_{n} \rightarrow u_{\infty} \text { in } L^{1}\left(\Omega ; \mathbb{R}^{m}\right) \quad \text { and } \quad \int_{\Omega} \operatorname{dist}\left(\nabla u_{n}(x), K\right) \mathrm{d} x \rightarrow 0 .
$$

Then $u_{n} \rightarrow u_{\infty}$ in $W^{1,1}$ and there exists $\left\{v_{n}\right\}_{n \in \mathbb{N}} \subset W^{1, \infty}\left(\Omega ; \mathbb{R}^{m}\right)$ such that

$$
\left\{\begin{array}{l}
v_{n}=u_{\infty} \text { on } \partial \Omega \\
\left|\left\{x \in \Omega: \nabla u_{n}(x) \neq \nabla v_{n}(x)\right\}\right| \rightarrow 0 \\
\left\|\operatorname{dist}\left(\nabla v_{n}, K\right)\right\|_{\infty, \Omega} \rightarrow 0
\end{array}\right.
$$

Lemma 2.8. Let $r>0$. Let $\rho>0$ be such that $\rho \bar{B} \subset \operatorname{int} C$, with $\bar{B}=\left\{\xi \in \mathbb{M}^{m \times d}:|\xi| \leq 1\right\}$. Then

$$
\left\{\xi \in \mathbb{M}^{m \times d}: \operatorname{dist}(\xi, \bar{C}) \leq \rho \frac{r}{2}\right\} \subset(1+r) \operatorname{int} C .
$$

Proof. Note that by l.s.p. and the continuity of the norm we have $\operatorname{dist}(\cdot, \operatorname{int} C)=\operatorname{dist}(\cdot, \bar{C})$. Let $r>0$. Let $\xi \in \mathbb{M}^{m \times d}$ be such that $\operatorname{dist}(\xi, \operatorname{int} C) \leq \rho \frac{r}{2}$. There exists $F_{r} \in \operatorname{int} C$ such that $\rho \frac{r}{2} \geq \operatorname{dist}(\xi, \operatorname{int} C) \geq\left|\xi-F_{r}\right|-\rho \frac{r}{2}$. Now, if we write $\xi=\xi-F_{r}+F_{r}$ then $\xi \in r \rho \bar{B}+\operatorname{int} C \subset(1+r) \operatorname{int} C$. 


\section{Proof of Theorem 1.1}

The following lemma gives a simplified formula for $\widehat{\mathcal{Z f}}$ under $\left(\mathscr{H}_{0}\right),\left(\mathscr{H}_{1}\right)$ and $\left(\mathscr{H}_{2}\right)$.

Lemma 3.1. Assume that $\left(\mathscr{H}_{0}\right),\left(\mathscr{H}_{1}\right)$ and $\left(\mathscr{H}_{2}\right)$ hold. Then

$$
\widehat{\mathcal{Z f}}(\xi)= \begin{cases}\mathcal{Z} f(\xi) & \text { if } \xi \in \operatorname{int} C \\ +\infty & \text { otherwise. }\end{cases}
$$

Proof. Let $\xi \in \partial C$. Lemma 2.1 (iv) and $\left(\mathscr{H}_{2}\right)$ give an increasing sequence $\left\{t_{n}\right\}_{n \geq 1} \subset\left[0,1\left[\right.\right.$ such that $t_{n} \rightarrow 1$ as $n \rightarrow+\infty$ and $\inf \left\{\mathcal{Z} f(\xi): \xi \in \bar{C} \backslash t_{n} \bar{C}\right\} \geq n$ for all $n \in \mathbb{N}^{*}$. Let $n \in \mathbb{N}^{*}$. By l.s.p., we have $t_{n+1} \xi \in t_{n+1} \bar{C} \backslash t_{n} \bar{C}$, since $t_{n}<t_{n+1}$. By Lemma 2.4, it follows that

$$
\widehat{\mathcal{Z} f}(\xi)=\lim _{n \rightarrow+\infty} \mathcal{Z} f\left(t_{n+1} \xi\right) \geq \liminf _{n \rightarrow+\infty} \inf \left\{\mathcal{Z} f(\xi): \xi \in \bar{C} \backslash t_{n} \bar{C}\right\} \geq \liminf _{n \rightarrow+\infty} n=+\infty .
$$

\section{Proof of Theorem 1.1}

We have to show that the function $\widehat{\mathcal{Z f}}$ is 1 -sup-quasiconvex, and

$$
\mathcal{Q} f=\widehat{\mathcal{Z} f}=\mathcal{Z} f .
$$

Consider the sequence $\left\{t_{n}\right\}_{n \in \mathbb{N}^{*}}$ given by Lemma 2.1 (iv). For each $n \in \mathbb{N}^{*}$, we set $f_{n}=\mathcal{Q} h_{n}$ where

$$
h_{n}(\xi)= \begin{cases}\mathcal{Z} f(\xi) & \text { if } \xi \in t_{n} \bar{C} \\ n(1+\operatorname{dist}(\xi, \bar{C})) & \text { if } \xi \in \mathbb{M}^{m \times d} \backslash t_{n} \bar{C} .\end{cases}
$$

By $\left(\mathscr{H}_{1}\right)$, it holds for every $\xi \in \mathbb{M}^{m \times d}$ and $n \in \mathbb{N}^{*}$

$$
f_{n}(\xi) \leq h_{n}(\xi) \leq \alpha_{n}(1+|\xi|)
$$

where $\alpha_{n}=\max \left\{\sup _{\xi \in t_{n} \bar{C}} f(\xi), 2 n(1+\operatorname{diam}(\bar{C}))\right\}<+\infty$, where $\operatorname{diam}(\bar{C})=\sup \{|\xi-\zeta|: \xi, \zeta \in \bar{C}\}$.

Let $n \in \mathbb{N}^{*}$, we will show that $f_{n} \leq f_{n+1}$. By l.s.p., we have $t_{n} \bar{C} \subset t_{n+1} \bar{C} \subset \operatorname{int} C$. Let $\xi \in \mathbb{M}^{m \times d}$,

- if $\xi \in t_{n} \bar{C}$ then $h_{n}(\xi)=h_{n+1}(\xi)=\mathcal{Z} f(\xi)$;

- if $\xi \in t_{n+1} \bar{C} \backslash t_{n} \bar{C}$ then, by Lemma 2.1 (iv), we obtain

$$
h_{n}(\xi)=n(1+\operatorname{dist}(\xi, \bar{C}))=n \leq \mathcal{Z} f(\xi)=h_{n+1}(\xi) ;
$$

- if $\xi \notin t_{n+1} \bar{C}$ then

$$
h_{n}(\xi)=n(1+\operatorname{dist}(\xi, \bar{C})) \leq(n+1)(1+\operatorname{dist}(\xi, \bar{C}))=h_{n+1}(\xi)
$$

Hence $h_{n} \leq h_{n+1}$ and then $f_{n} \leq f_{n+1}$. Note also that, by Lemma 2.2, we have $f_{n} \leq \mathcal{Z} f \leq f$. Thus, we have that $\left\{f_{n}\right\}_{n \in \mathbb{N}^{*}}$ is a nondecreasing sequence of quasiconvex functions satisfying

$$
f_{n}(\xi) \leq \alpha_{n}(1+|\xi|), \text { and } f_{n}(\xi) \leq \mathcal{Z} f(\xi) \leq f(\xi)
$$

for all $\xi \in \mathbb{M}^{m \times d}$ and $n \in \mathbb{N}^{*}$. Set $f_{\infty}=\sup _{n \in \mathbb{N}^{*}} f_{n}$, then, by the right hand side inequality in (3.1), it holds that $f_{\infty} \leq \widehat{\mathcal{Z f}}$. 
Now, we shall show that $f_{\infty} \geq \widehat{\mathcal{Z} f}$. Let $\xi \in \mathbb{M}^{m \times d}$ and $k \in \mathbb{N}^{*}$. Without loss of generality, we may assume that $f_{\infty}(\xi)<+\infty$. By Lemma A.2 it holds that for every $n \in \mathbb{N}^{*}$ there exists $\phi_{n}^{k} \in W_{0}^{1, \infty}\left(Y ; \mathbb{R}^{m}\right)$ such that

$$
\frac{1}{2 k}+f_{\infty}(\xi) \geq \int_{A_{n}^{k}} \mathcal{Z} f\left(\xi+\nabla \phi_{n}^{k}(x)\right) \mathrm{d} x+n \int_{Y \backslash A_{n}^{k}} \operatorname{dist}\left(\xi+\nabla \phi_{n}^{k}(x), \bar{C}\right) \mathrm{d} x+n\left|Y \backslash A_{n}^{k}\right|
$$

with $A_{n}^{k}=\left\{x \in Y: \xi+\nabla \phi_{n}^{k}(x) \in t_{n} \bar{C}\right\}$. We deduce that

$$
\begin{gathered}
\lim _{n \rightarrow+\infty} \int_{Y} \operatorname{dist}\left(\xi+\nabla \phi_{n}^{k}(x), \bar{C}\right) \mathrm{d} x=0 . \\
\lim _{n \rightarrow+\infty}\left|Y \backslash A_{n}^{k}\right|=0 .
\end{gathered}
$$

Since for any $\zeta \in \mathbb{M}^{m \times d}$ it holds that $|\zeta| \leq \operatorname{diam}(\bar{C})+\operatorname{dist}(\zeta, \bar{C}),(3.2)$ implies that $\left\{\xi+\nabla \phi_{n}^{k}\right\}_{n \in \mathbb{N}^{*}}$ is bounded in $L^{1}\left(Y ; \mathbb{M}^{m \times d}\right)$. Using Poincaré inequality and compact imbedding of $W^{1,1}\left(Y ; \mathbb{R}^{m}\right)$ in $L^{1}\left(Y ; \mathbb{R}^{m}\right)$ we deduce that there exists a subsequence (not relabelled) $\left\{\phi_{n}^{k}\right\}_{n \in \mathbb{N}^{*}}$ converging in $L^{1}$. Applying Lemma 2.7, we can find a sequence $\left\{\psi_{n}^{k}\right\}_{n \in \mathbb{N}^{*}} \subset W_{0}^{1, \infty}\left(Y ; \mathbb{R}^{m}\right)$ such that

$$
\begin{gathered}
\lim _{n \rightarrow+\infty}\left|\left\{x \in Y: \nabla \phi_{n}^{k}(x) \neq \nabla \psi_{n}^{k}(x)\right\}\right|=0, \text { and } \\
\lim _{n \rightarrow+\infty}\left\|\operatorname{dist}\left(\xi+\nabla \psi_{n}^{k}, \bar{C}\right)\right\|_{\infty, Y}=0 .
\end{gathered}
$$

Let $\rho>0$ be such that $\rho \bar{B} \subset \operatorname{int} C$, where $\bar{B}=\left\{\xi \in \mathbb{M}^{m \times d}:|\xi| \leq 1\right\}$. By (3.5), there exists $\sigma(k) \in \mathbb{N}^{*}$ such that for every $n \geq \sigma(k)$

$$
\left\|\operatorname{dist}\left(\xi+\nabla \psi_{n}^{k}, \bar{C}\right)\right\|_{\infty, Y} \leq \frac{\rho}{2 k}
$$

We construct $\sigma: \mathbb{N}^{*} \rightarrow \mathbb{N}^{*}$ in order to obtain an increasing function of $k$. By Lemma 2.8, we deduce $\xi+\nabla \psi_{n}^{k}(x) \in$ $\left(1+\frac{1}{k}\right)$ int $C$ a.e. in $Y$ for all $n \geq \sigma(k)$.

For each $l \in \mathbb{N}^{*}$ we denote by $M_{l}=\sup \left\{f(\zeta): \zeta \in t_{l} \bar{C}\right\}$ which is finite since Lemma 2.1 (iii). By (3.3) and (3.4), there exists $\delta(k) \geq 1$ such that for every $n \geq \delta(k)$

$$
\max \left\{\left|\left\{x \in Y: \nabla \phi_{n}^{k}(x) \neq \nabla \psi_{n}^{k}(x)\right\}\right|,\left|Y \backslash A_{n}^{k}\right|\right\} \leq \frac{1}{4 k M_{\sigma(k)}} .
$$

Now, we take $n \geq \max \{\delta(k), \sigma(k)\}$ then

$$
\max \left\{\left|B_{n}^{k}\right|,\left|Y \backslash A_{n}^{k}\right|\right\} \leq \frac{1}{4 k M_{\sigma(k)}} \quad \text { and } \quad \tau_{k}\left(\xi+\nabla \psi_{n}^{k}(x)\right) \in \operatorname{int} C \text { a.e. in } Y,
$$

where $B_{n}^{k}=\left|\left\{x \in Y: \nabla \phi_{n}^{k}(x) \neq \nabla \psi_{n}^{k}(x)\right\}\right|$ and $\tau_{k}=\left(1+k^{-1}\right)^{-1}$. Set $G_{n}^{k}=B_{n}^{k} \cup\left(Y \backslash\left(B_{n}^{k} \cup A_{n}^{k}\right)\right)$, we have that $\left|G_{n}^{k}\right| \leq\left(2 k M_{\sigma(k)}\right)^{-1}$ since $B_{n}^{k} \cup A_{n}^{k} \supset A_{n}^{k}$. Then it holds

$$
\begin{aligned}
\int_{Y} \mathcal{Z} f\left(t_{\sigma(k)} \tau_{k}\left(\xi+\nabla \psi_{n}^{k}(x)\right)\right) \mathrm{d} x= & \int_{G_{n}^{k}} \mathcal{Z} f\left(t_{\sigma(k)} \tau_{k}\left(\xi+\nabla \psi_{n}^{k}(x)\right)\right) \mathrm{d} x \\
& +\int_{\left(Y \backslash B_{n}^{k}\right) \cap A_{n}^{k}} \mathcal{Z} f\left(t_{\sigma(k)} \tau_{k}\left(\xi+\nabla \psi_{n}^{k}(x)\right)\right) \mathrm{d} x \\
\leq & \left|G_{k}\right| \sup _{\zeta \in t_{\sigma(k)} \bar{C}} \mathcal{Z} f(\zeta)+\int_{A_{n}^{k}} \mathcal{Z} f\left(t_{\sigma(k)} \tau_{k}\left(\xi+\nabla \phi_{n}^{k}(x)\right)\right) \mathrm{d} x \\
\leq & \frac{1}{2 k}+\int_{A_{n}^{k}} \mathcal{Z} f\left(t_{\sigma(k)} \tau_{k}\left(\xi+\nabla \phi_{n}^{k}(x)\right)\right) \mathrm{d} x .
\end{aligned}
$$


By convexity of the distance function, we deduce from (3.2) that $\xi \in \bar{C}$. The l.s.p. implies that $t_{\sigma(k)} \tau_{k} \xi \in \operatorname{int} C$. Using Lemma 2.6, we deduce that for every $k \in \mathbb{N}^{*}$ and every $n \geq \max \{\delta(k), \sigma(k)\}$

$$
\mathcal{Z} f\left(t_{\sigma(k)} \tau_{k} \xi\right) \leq \frac{1}{2 k}+\int_{A_{n}^{k}} \mathcal{Z} f\left(t_{\sigma(k)} \tau_{k}\left(\xi+\nabla \phi_{n}^{k}(x)\right)\right) \mathrm{d} x,
$$

with $A_{n}^{k}=\left\{x \in Y: \xi+\nabla \phi_{n}^{k}(x) \in t_{n} \bar{C}\right\}$.

Let $s \in \mathbb{N}^{*}$. By $\left(\mathscr{H}_{0}\right)$, there exists $\eta_{s}>0$ such that for every $t \in\left[0,1\left[\right.\right.$ and $\zeta \in \operatorname{int} C$ if $1-t \leq \eta_{s}$ then $\mathcal{Z} f(t \zeta) \leq \mathcal{Z} f(\zeta)+\frac{1}{s}$. There also exists an integer $k_{s} \geq 1$ such that $1-t_{\sigma(k)} \tau_{k} \leq \eta_{s}$ for all $k \geq k_{s}$ since $\sigma$ is increasing. Thus, if we take $k \geq k_{s}$ then for every $n \geq \max \{\sigma(k), \delta(k)\}$

$$
\begin{aligned}
\int_{A_{n}^{k}} \mathcal{Z} f\left(t_{\sigma(k)} \tau_{k}\left(\xi+\nabla \phi_{n}^{k}(x)\right)\right) \mathrm{d} x & \leq \frac{1}{s}+\int_{A_{n}^{k}} \mathcal{Z} f\left(\xi+\nabla \phi_{n}^{k}(x)\right) \mathrm{d} x \\
& \leq \frac{1}{s}+\frac{1}{2 k}+f_{\infty}(\xi) .
\end{aligned}
$$

Hence, by (3.6)

By Lemmas 2.3 and 2.4, it follows

$$
\mathcal{Z} f\left(t_{\sigma(k)} \tau_{k} \xi\right) \leq \frac{1}{s}+\frac{1}{k}+f_{\infty}(\xi)
$$

$$
\widehat{\mathcal{Z f}}(\xi)=\lim _{k \rightarrow+\infty} \mathcal{Z} f\left(t_{\sigma(k)} \tau_{k} \xi\right) \leq \frac{1}{s}+f_{\infty}(\xi) .
$$

Letting $s \rightarrow+\infty$, we obtain $\widehat{\mathcal{Z} f} \leq f_{\infty}$.

Now, by (3.1), (1.1) and Lemma 3.1, it follows

$$
\mathcal{Q} f \leq \mathcal{Z} f \leq \widehat{\mathcal{Z} f}=f^{\infty} \leq \mathcal{Q}(\mathcal{Z} f) \leq \mathcal{Q} f .
$$

The proof is complete.

\section{Proof of Theorem 1.3}

The proof follows essentially the same lines as the proof of Theorem 1.1, however it is worth to write it here because of some differences due to the behavior of $\mathcal{Z} f$ near the boundary of $C$.

We divide the proof into two steps. In the first step we show that $\widehat{\mathcal{Z f}}$ is 1 -sup-quasiconvex. In the second step we show the representation formula for $\mathcal{Q} f$.

Step 1. Assume without loss of generality that $\mathcal{Z} f \not \equiv 0$ in int $C$. For each $n \in \mathbb{N}$, we set $f_{n}=\mathcal{Q} g_{n}$ where

$$
g_{n}(\xi)= \begin{cases}\mathcal{Z} f(\xi) & \text { if } \xi \in \operatorname{int} C \\ n(1+\operatorname{dist}(\xi, \bar{C})) & \text { if } \xi \in \mathbb{M}^{m \times d} \backslash \operatorname{int} C .\end{cases}
$$

Set $M=\sup _{\xi \in \operatorname{int} C} \mathcal{Z} f(\xi)$ which is finite since $\left(\mathscr{H}_{3}\right)$. We have that $\left\{f_{n}\right\}_{n \in \mathbb{N}}$ is a nondecreasing sequence of quasiconvex functions satisfying

$$
f_{n}(\xi) \leq \max (M, 2 n(1+\operatorname{diam}(\bar{C})))(1+|\xi|)
$$

for all $\xi \in \mathbb{M}^{m \times d}$ and $n \in \mathbb{N}$. For each $n \in \mathbb{N}^{*}$ we have $f_{n} \leq \mathcal{Z} f \leq f$ in $\operatorname{int} C$, and $f_{n} \leq \widehat{\mathcal{Z f}} \leq f$ in $\bar{C}$. Indeed, let $\xi \in \partial C$, by Lemma 2.4 there exists a sequence $\left\{t_{k}\right\}_{k \in \mathbb{N}^{*}} \subset\left[0,1\left[\right.\right.$ such that $t_{k} \rightarrow 1$ and $\lim _{k \rightarrow+\infty} \mathcal{Z} f\left(t_{k} \xi\right)=$ 
$\lim _{[0,1[\ni t \rightarrow 1} \widehat{\mathcal{Z f}}(t \xi)$. By l.s.p., $t_{k} \xi \in \operatorname{int} C$ for all $k \in \mathbb{N}^{*}$. Thus, by $\left(\mathscr{H}_{4}\right)$, we obtain for all $n \in \mathbb{N}^{*}$

$$
f_{n}(\xi) \leq \lim _{k \rightarrow+\infty} \mathcal{Z} f\left(t_{k} \xi\right)=\widehat{\mathcal{Z} f}(\xi) \leq \liminf _{k \rightarrow+\infty} f\left(t_{k} \xi\right) \leq f(\xi)
$$

And so for every $\xi \in \mathbb{M}^{m \times d}$ and $n \in \mathbb{N}^{*}$

$$
f_{n}(\xi) \leq f_{\infty}(\xi) \leq \widehat{\mathcal{Z f}}(\xi) \leq f(\xi)
$$

where $f_{\infty}=\sup _{n \geq 1} f_{n}$.

Now, we shall show that $f_{\infty} \geq \widehat{\mathcal{Z} f}$. Let $\xi \in \mathbb{M}^{m \times d}$ and $k \in \mathbb{N}^{*}$. Without loss of generality, we may assume that $f_{\infty}(\xi)<+\infty$. By Lemma A.2 it holds that for every $n \in \mathbb{N}$ there exists $\phi_{n}^{k} \in W_{0}^{1, \infty}\left(Y ; \mathbb{R}^{m}\right)$ such that

$$
\frac{1}{2 k}+f_{\infty}(\xi) \geq \int_{A_{n}^{k}} \mathcal{Z} f\left(\xi+\nabla \phi_{n}^{k}(x)\right) \mathrm{d} x+n \int_{Y \backslash A_{n}^{k}} \operatorname{dist}\left(\xi+\nabla \phi_{n}^{k}(x), \bar{C}\right) \mathrm{d} x+n\left|Y \backslash A_{n}^{k}\right|
$$

with $A_{n}^{k}=\left\{x \in Y: \xi+\nabla \phi_{n}^{k}(x) \in \operatorname{int} C\right\}$. We deduce that

$$
\begin{gathered}
\lim _{n \rightarrow+\infty} \int_{Y} \operatorname{dist}\left(\xi+\nabla \phi_{n}^{k}(x), \bar{C}\right) \mathrm{d} x=0 . \\
\lim _{n \rightarrow+\infty}\left|Y \backslash A_{n}^{k}\right|=0 .
\end{gathered}
$$

Since for any $\zeta \in \mathbb{M}^{m \times d}$ it holds that $|\zeta| \leq \operatorname{diam}(\bar{C})+\operatorname{dist}(\zeta, \bar{C}),(4.2)$ implies that $\left\{\xi+\nabla \phi_{n}^{k}\right\}_{n \in \mathbb{N}}$ is bounded in $L^{1}\left(Y ; \mathbb{M}^{m \times d}\right)$. Using Poincaré inequality and compact imbedding of $W^{1,1}\left(Y ; \mathbb{R}^{m}\right)$ in $L^{1}\left(Y ; \mathbb{R}^{m}\right)$ we deduce that there exists a subsequence (not relabelled) $\left\{\phi_{n}^{k}\right\}_{n \in \mathbb{N}}$ converging in $L^{1}$. Applying Lemma 2.7, we can find a sequence $\left\{\psi_{n}^{k}\right\}_{n \in \mathbb{N}} \subset W_{0}^{1, \infty}\left(Y ; \mathbb{R}^{m}\right)$ such that

$$
\begin{gathered}
\lim _{n \rightarrow+\infty}\left|\left\{x \in Y: \nabla \phi_{n}^{k}(x) \neq \nabla \psi_{n}^{k}(x)\right\}\right|=0 \quad \text { and } \\
\lim _{n \rightarrow+\infty}\left\|\operatorname{dist}\left(\xi+\nabla \psi_{n}^{k}(x), \bar{C}\right)\right\|_{\infty, Y}=0 .
\end{gathered}
$$

As in the proof of Theorem 1.1 we deduce that there exists an increasing sequence $\mathbb{N}^{*} \ni l \mapsto \sigma(l) \in \mathbb{N}^{*}$ such that

$$
\begin{gathered}
\max \left\{\left|\left\{x \in Y: \nabla \phi_{\sigma(k)}^{k}(x) \neq \nabla \psi_{\sigma(k)}^{k}(x)\right\}\right|,\left|Y \backslash A_{\sigma(k)}^{k}\right|\right\} \leq \frac{1}{4 k M} \\
\tau_{k}\left(\xi+\nabla \psi_{\sigma(k)}^{k}(x)\right) \in \operatorname{int} C \text { a.e. in } Y,
\end{gathered}
$$

where $\tau_{k}=\left(1+k^{-1}\right)^{-1}$. To simplify notation we write $\phi_{k}, \psi_{k}$ and $A_{k}$ for, respectively, $\phi_{\sigma(k)}^{k}, \psi_{\sigma(k)}^{k}$ and $A_{\sigma(k)}^{k}$. Set $B_{k}=\left\{x \in Y: \nabla \psi_{k}(x) \neq \nabla \phi_{k}(x)\right\}$ and $G_{k}=B_{k} \cup\left(Y \backslash\left(B_{k} \cup A_{k}\right)\right)$. By $\left(\mathscr{H}_{3}\right)$ we have

$$
\begin{aligned}
\int_{Y} \mathcal{Z} f\left(\tau_{k}\left(\xi+\nabla \psi_{k}\right)\right) \mathrm{d} x & \leq \int_{G_{k}} \mathcal{Z} f\left(\tau_{k}\left(\xi+\nabla \psi_{k}\right)\right) \mathrm{d} x+\int_{A_{k}} \mathcal{Z} f\left(\tau_{k}\left(\xi+\nabla \phi_{k}\right)\right) \mathrm{d} x \\
& \leq \frac{1}{2 k}+\int_{A_{k}} \mathcal{Z} f\left(\tau_{k}\left(\xi+\nabla \phi_{k}\right)\right) \mathrm{d} x
\end{aligned}
$$

Let $s \in \mathbb{N}^{*}$. By $\left(\mathscr{H}_{0}\right)$, there exists $\eta_{s}>0$ such that for every $t \in\left[0,1\left[\right.\right.$ and $\xi \in \operatorname{int} C$ if $1-t \leq \eta_{s}$ then $\mathcal{Z} f(t \xi) \leq \mathcal{Z} f(\xi)+\frac{1}{s}$. Consider $k \in \mathbb{N}^{*}$ such that $k+1>\left(\eta_{s}\right)^{-1}$, it follows that

$$
\begin{aligned}
\int_{A_{k}} \mathcal{Z} f\left(\tau_{k}\left(\xi+\nabla \phi_{k}(x)\right)\right) \mathrm{d} x & \leq \frac{1}{s}+\int_{A_{k}} \mathcal{Z} f\left(\xi+\nabla \phi_{k}(x)\right) \mathrm{d} x \\
& \leq \frac{1}{s}+\frac{1}{2 k}+f_{\infty}(\xi),
\end{aligned}
$$


and then

$$
\int_{Y} \mathcal{Z} f\left(\tau_{k}\left(\xi+\nabla \psi_{k}(x)\right)\right) \mathrm{d} x \leq \frac{1}{s}+\frac{1}{k}+f_{\infty}(\xi)
$$

By convexity of the distance function, we deduce from (4.2) that $\xi \in \bar{C}$. The l.s.p. implies that $\tau_{k} \xi \in \operatorname{int} C$. According to Lemma 2.6 together with Lemmas 2.4 and 2.3, we deduce from (4.4) that

$$
\widehat{\mathcal{Z} f}(\xi) \leq \liminf _{k \rightarrow+\infty} \mathcal{Z} f\left(\tau_{k} \xi\right) \leq \frac{1}{s}+f_{\infty}(\xi)
$$

Letting $s \rightarrow+\infty$, we obtain $\widehat{\mathcal{Z f}} \leq f_{\infty}$, which finishes the proof of the first step.

Step 2. We shall show that

$$
\mathcal{Q} f=\widehat{\mathcal{Z} f}=\overline{\mathcal{Z} f} \text {. }
$$

We have that $\mathcal{Q} f \leq \mathcal{Z} f$ since $\mathcal{Q} f$ satisfies inequality (1.1). Now, let $\xi \in \partial C$, by lower semicontinuity of $\mathcal{Q} f$ we have

$$
\widehat{\mathcal{Z} f}(\xi)=\lim _{[0,1[\ni t \rightarrow 1} \mathcal{Z} f(t \xi) \geq \liminf _{[0,1[\ni t \rightarrow 1} \mathcal{Q} f(t \xi) \geq \mathcal{Q} f(\xi) .
$$

We deduce that $\mathcal{Q} f \leq \widehat{\mathcal{Z} f}$. By (4.1) we have $\widehat{\mathcal{Z f}}=f_{\infty} \leq \mathcal{Q} f$. Now, it holds $\widehat{\mathcal{Z f}} \geq \overline{\mathcal{Z} f}$ on $\bar{C}$, and by definition of $\widehat{\mathcal{Z} f}$, we obtain $\widehat{\mathcal{Z} f} \geq \overline{\mathcal{Z} f}$. Since $\mathcal{Q} f \leq \mathcal{Z} f$, it follows that $\mathcal{Q} f \leq \overline{\mathcal{Z} f}$. Thus, we obtain

$$
\mathcal{Q} f \leq \overline{\mathcal{Z} f} \leq \widehat{\mathcal{Z f}}=f_{\infty} \leq \mathcal{Q} f
$$

which finishes the proof.

\section{Upper bound And approximation of Sobolev functions Via Continuous PIECEWISE AFFINE FUNCTIONS}

\subsection{Upper bound for continuous piecewise affine functions}

Let us denote by $\operatorname{Aff}\left(\Omega ; \mathbb{R}^{m}\right)$ the space of all continuous piecewise affine functions from $\Omega$ to $\mathbb{R}^{m}$, i.e., $u \in \operatorname{Aff}\left(\Omega ; \mathbb{R}^{m}\right)$ if and only if $u$ is continuous and there exists a finite family $\left\{U_{i}\right\}_{i \in I}$ of open disjoint subsets of $U$ such that $\left|U \backslash \cup_{i \in I} U_{i}\right|=0$ and for every $i \in I, \nabla u(x)=\xi_{i}$ in $U_{i}$ with $\xi_{i} \in \mathbb{M}^{m \times d}$. The proof of the following lemma follows by an easy adaptation of Lemma 3.1 in [3].

Lemma 5.1. Let $p \in[1,+\infty]$. Let $g: \mathbb{M}^{m \times d} \rightarrow[0,+\infty]$ be a Borel measurable function and $U \subset \mathbb{R}^{d}$ be a bounded open set. Then for every $u \in \operatorname{Aff}\left(U ; \mathbb{R}^{m}\right)$

$$
\inf \left\{\liminf _{n \rightarrow+\infty} \int_{U} g\left(\nabla u_{n}\right) \mathrm{d} x: u+W_{0}^{1, \infty}\left(U ; \mathbb{R}^{m}\right) \ni u_{n} \rightarrow u \text { in } W^{1, p}\right\} \leq \int_{U} \mathcal{Z} g(\nabla u) \mathrm{d} x
$$

(replace “- by "**” when $p=+\infty)$.

\subsection{Approximation results}

Let $|\cdot|_{1, p, A}\left(\right.$ resp. $\left.|\cdot|_{p, A}\right)$ stand for the norm of $W^{1, p}\left(A ; \mathbb{R}^{m}\right)\left(\right.$ resp. $\left.L^{p}\left(A ; \mathbb{R}^{m}\right)\right)$ where $A \subset \mathbb{R}^{d}$ is open and $p \in[1,+\infty]$. Let $B=\left\{\xi \in \mathbb{M}^{m \times d}:|\xi|<1\right\}$ be the unit ball of $\mathbb{M}^{m \times d}$.

The proof of the following lemma is omitted since it is a particular case of Theorem 10.16 in [9]. 
Lemma 5.2. Let $\Omega \subset \mathbb{R}^{d}$ be a bounded open set. Let $K \subset \mathbb{M}^{m \times d}$ be a compact set and $u \in \mathcal{C}^{1}\left(\bar{\Omega} ; \mathbb{R}^{m}\right)$ such that

$$
\forall x \in \bar{\Omega} \quad \nabla u(x) \in \operatorname{int} K .
$$

Then for every $l \in \mathbb{N}^{*}$ there exists $u_{l} \in W^{1, \infty}\left(\Omega ; \mathbb{R}^{m}\right)$ and an open set $\Omega_{l} \subset \Omega$ such that

(i) $u_{l}\left\lfloor\Omega_{l} \in \operatorname{Aff}\left(\Omega_{l} ; \mathbb{R}^{m}\right)\right.$ and $u_{l}=u$ near $\partial \Omega$;

(ii) $\nabla u_{l}(x) \in \operatorname{int} K$ a.e. in $\Omega$;

(iii) $\left\|u_{l}-u\right\|_{1, \infty, \Omega} \leq \frac{1}{l}$;

(iv) $\left|\Omega \backslash \Omega_{l}\right| \leq \frac{1}{l}$ and $\left|\partial \Omega_{l}\right|=0$.

To prove the following approximation result, we follow the arguments of Corollary 10.21 in [9]. We pretend no originality here in doing this, we just make an adaptation in our framework.

Proposition 5.1. Let $K \subset \mathbb{M}^{m \times d}$ be a compact convex set with $0 \in \operatorname{int} K$. Let $t \in\left[0,1\left[\right.\right.$ and $u \in W^{1, \infty}\left(\Omega ; \mathbb{R}^{m}\right)$ be such that

$$
\nabla u(x) \in t K \text { a.e. in } \Omega \text {. }
$$

Then for every integer $n>\frac{1}{1-t}$ there exist $u_{n} \in W^{1, \infty}\left(\Omega ; \mathbb{R}^{m}\right)$ and an open set $\Omega_{n} \subset \Omega$ such that

(i) $u_{n}\left\lfloor\Omega_{n} \in \operatorname{Aff}\left(\Omega_{n} ; \mathbb{R}^{m}\right)\right.$ and $u_{n}=u$ on $\partial \Omega$;

(ii) $\left\|u_{n}-u\right\|_{1, p, \Omega} \leq n^{-1}$ for all $p \in[1,+\infty[$.

(iii) $\nabla u_{n}(x) \in\left(t+\frac{1}{n}\right) K$ a.e. in $\Omega$;

(iv) $\left|\Omega \backslash \Omega_{n}\right| \leq \frac{1}{n}$ and $\left|\partial \Omega_{n}\right|=0$.

Proof. Since $0 \in \operatorname{int} K$, there exists $r>0$ such that $r \bar{B} \subset \operatorname{int} K$. Let $t \in\left[0,1\left[\right.\right.$ and $u \in W^{1, \infty}\left(\Omega ; \mathbb{R}^{m}\right)$ be such that $\nabla u(x) \in t K$ a.e. in $\Omega$. Fix $s \in \mathbb{N}^{*}$. Let $\sigma: \mathbb{N}^{*} \rightarrow \mathbb{N}^{*}$ be an increasing function such that the set $U_{\sigma(s)}=\left\{x \in \Omega: \operatorname{dist}\left(x ; \mathbb{R}^{d} \backslash \Omega\right)>\sigma(s)^{-1}\right\}$ satisfies $\left|\Omega \backslash U_{\sigma(s)}\right| \leq \frac{1}{s}$. Let us define $\psi_{s} \in \mathcal{C}_{0}^{1}(\Omega ;[0,1])$ by

$$
\psi_{s}(x)=\left\{\begin{array}{ll}
1 & \text { if } x \in \bar{U}_{2-1} \sigma(s) \\
0 & \text { if } x \in \Omega \backslash U_{\sigma(s)}
\end{array} .\right.
$$

To simplify notation we write $U_{s}$ instead of $U_{\sigma(s)}$. Set $\nu_{s}=\left\|\nabla \psi_{s}\right\|_{\infty}$, we can assume that there exists $c_{0}>0$ such that $\nu_{s} \geq c_{0}$ for all $s \in \mathbb{N}^{*}$.

By Sobolev imbedding theorem, we can assume that $u$ is continuous in $\bar{\Omega}$. For every $k>2 \sigma(s)$, consider $\rho_{k}$ a smooth mollifier with support in the ball $B_{\frac{1}{k}}(0) \subset \mathbb{R}^{d}$. Then the function $v_{k}=\rho_{k} \star\left(u\left\lfloor_{U_{s}}\right) \in \mathcal{C}_{0}^{\infty}\left(\Omega ; \mathbb{R}^{m}\right)\right.$ satisfies

$$
\begin{aligned}
& -\nabla v_{k}(x) \in t K \text { for every } x \in \bar{U}_{s} \\
& \text { - }\left\|v_{k}-u\right\|_{1, p, U_{s}} \leq \frac{1}{2 s \nu_{s}} \text { for all } p \in[1,+\infty[; \\
& \text { - }\left\|v_{k}-u\right\|_{\infty, U_{s}} \leq \frac{r}{2 s \nu_{s}}
\end{aligned}
$$

for all $k \geq \hat{k}(s)=\max \{k(s), 2 \sigma(s)\}$ for some $k(s) \in \mathbb{N}^{*}$.

By l.s.p., we have $t K \subset \operatorname{int} K$. By Lemma 5.2 , for every $k \geq \hat{k}(s)$ there exist a sequence $\left\{v_{l, k}\right\}_{l \in \mathbb{N}^{*}} \subset$ $W^{1, \infty}\left(U_{s} ; \mathbb{R}^{m}\right)$ and a sequence of open sets $\left\{U_{l, k}\right\}_{l \in \mathbb{N}^{*}} \subset U_{s}$ satisfying for every $l \in \mathbb{N}^{*}$

- $v_{l, k}\left\lfloor_{U_{l, k}} \in \operatorname{Aff}\left(U_{l, k} ; \mathbb{R}^{m}\right)\right.$ and $v_{l, k}=v_{k}$ near $\partial U_{s} ;$

- $\nabla v_{l, k}(x) \in \operatorname{int} K$ a.e. in $U_{s}$;

- $\left\|v_{l, k}-v_{k}\right\|_{1, \infty, U_{s}} \leq \frac{r}{l}$

$-\left|U_{s} \backslash U_{l, k}\right| \leq \frac{r}{l}$ and $\left|\partial U_{l, k}\right|=0$. 
It is easy to see that

$$
\nabla v_{l, k}(x) \in t K+\frac{r}{l} \bar{B} \text { a.e. in } U_{s}
$$

for all $l \in \mathbb{N}^{*}$ and $k \geq \hat{k}(s)$. We define

$$
u_{l, k}(x)= \begin{cases}\psi_{s}(x) v_{l, k}(x)+\left(1-\psi_{s}(x)\right) u(x) & \text { if } x \in U_{s} \\ u(x) & \text { if } x \in \Omega \backslash U_{s} .\end{cases}
$$

We have that $u_{l, k} \in W^{1, \infty}\left(\Omega ; \mathbb{R}^{m}\right), u_{l, k}=u$ on $\partial \Omega$ and

$$
\nabla u_{l, k}(x)=\psi_{s}(x) \nabla v_{l, k}(x)+\left(1-\psi_{s}(x)\right) \nabla u(x)+\nabla \psi_{s}(x) \otimes\left(v_{l, k}(x)-u(x)\right) \text { a.e. in } U_{s} \text {. }
$$

Let $\hat{l}(s) \geq 2 s \nu_{s}$ be an integer. If $l \geq \hat{l}(s)$ and $k \geq \hat{k}(s)$ then

$$
\left\|\nabla \psi_{s} \otimes\left(v_{l, k}-u\right)\right\|_{\infty, U_{s}} \leq \frac{r}{s}
$$

Set $u_{s}=u_{\hat{l}(s), \hat{k}(s)}$ and $\Omega_{s}=U_{\hat{l}(s), \hat{k}(s)}$. Then $u_{s}\left\lfloor\Omega_{s} \in \operatorname{Aff}\left(\Omega_{s} ; \mathbb{R}^{m}\right)\right.$ with $\left|\Omega \backslash \Omega_{s}\right| \leq s^{-1}+\left(2 s c_{0}\right)^{-1}$ and

$$
\nabla u_{s}(x) \in t K+\left(\frac{1}{s}+\frac{1}{2 s c_{0}}\right) r \bar{B} \text { a.e. in } \Omega .
$$

Set $v_{s}=v_{\hat{l}(s), \hat{k}(s)}$, we have for every $p \in[1,+\infty[$

$$
\begin{aligned}
& \left\|u_{s}-u\right\|_{1, p, \Omega}=\left\|u_{s}-u\right\|_{1, p, U_{s}} \leq\left\|v_{s}-u\right\|_{1, p, U_{s}} \\
& \leq\left\|v_{s}-v_{\hat{k}(s)}\right\|_{1, p, U_{s}}+\left\|v_{\hat{k}(s)}-u\right\|_{1, p, U_{s}} \\
& \leq 2^{\frac{1}{p}}|\Omega|^{\frac{1}{p}}\left\|v_{s}-v_{\hat{k}(s)}\right\|_{1, \infty, U_{s}}+\left\|v_{\hat{k}(s)}-u\right\|_{1, p, U_{s}} \\
& \leq \frac{1}{2 s \nu_{s}}\left(1+r 2^{\frac{1}{p}}|\Omega|^{\frac{1}{p}}\right) \text {. }
\end{aligned}
$$

Let $n>\frac{1}{1-t}$. Choose $s(n) \in \mathbb{N}^{*}$ such that

$$
\frac{1}{2 s(n) c_{0}}\left(1+r 2^{\frac{1}{p}}|\Omega|^{\frac{1}{p}}+2\left(r+c_{0}\right)\right) \leq \frac{1}{n}
$$

and set $u_{n}=u_{s(n)}$ and $\Omega_{n}=\Omega_{s(n)}$. Then (i), (ii), (iii) and (iv) are satisfied.

\section{Proofs of Theorems 1.2 And 1.4}

Lemma 6.1. Assume that $\left(\mathscr{H}_{1}\right)$ holds. Let $u \in W^{1, \infty}\left(\Omega ; \mathbb{R}^{m}\right)$ and $t \in[0,1[$ such that

$$
\nabla u(x) \in t \bar{C} \text { a.e. in } \Omega \text {. }
$$

Then there exists a sequence $\left\{u_{n}\right\}_{n \in \mathbb{N}} \subset u+W_{0}^{1, \infty}\left(\Omega ; \mathbb{R}^{m}\right)$ such that

$$
u_{n} \rightarrow u \text { in } L^{1} \text { and } \limsup _{n \rightarrow+\infty} \int_{\Omega} f\left(\nabla u_{n}(x)\right) \mathrm{d} x \leq \int_{\Omega} \mathcal{Z} f(\nabla u(x)) \mathrm{d} x
$$


Proof. Let $u \in W^{1, \infty}\left(\Omega ; \mathbb{R}^{m}\right)$ and $t \in[0,1[$ such that $\nabla u(x) \in t \bar{C}$ a.e. in $\Omega$. By Proposition 5.1 there exist $\left\{u_{n}\right\}_{n \in \mathbb{N}^{*}} \subset W^{1, \infty}\left(\Omega ; \mathbb{R}^{m}\right)$ and a sequence of open sets $\left\{\Omega_{n}\right\}_{n \in \mathbb{N}^{*}}, \Omega_{n} \subset \Omega$ such that for every integer $n>$ $(1-t)^{-1}$

- $u_{n}\left\lfloor\Omega_{n} \in \operatorname{Aff}\left(\Omega_{n} ; \mathbb{R}^{m}\right)\right.$ and $u_{n}=u$ on $\partial \Omega$;

- $\left\|u_{n}-u\right\|_{1, p, \Omega} \leq n^{-1}$ for all $p \in[1,+\infty[;$

- $\nabla u_{n}(x) \in\left(t+n^{-1}\right) \bar{C}$ a.e. in $\Omega$;

$-\left|\Omega \backslash \Omega_{n}\right| \leq n^{-1}$ and $\left|\partial \Omega_{n}\right|=0$.

We can assume, up to a subsequence, that $\nabla u_{n}(\cdot) \rightarrow \nabla u(\cdot)$ a.e. in $\Omega$ and $u_{n} \rightarrow u$ in $L^{1}$ as $n \rightarrow+\infty$. Choose $n_{t} \in \mathbb{N}^{*}$ in order to have

$$
t+\frac{1}{n_{t}}<\frac{1+t}{2}<1
$$

Let $n \geq n_{t}$, then $\nabla u_{n}(x) \in \frac{1+t}{2} \bar{C}$ a.e. in $\Omega$. Set $M_{t}=\sup \left\{f(\xi): \xi \in \frac{1+t}{2} \bar{C}\right\}$ which is finite since $\left(\mathscr{H}_{1}\right)$.

By Lemma 5.1, for every $n \geq n_{t}$ there exists $\left\{v_{m}^{n}\right\}_{m \in \mathbb{N}^{*}} \subset u_{n}+W_{0}^{1, \infty}\left(\Omega_{n} ; \mathbb{R}^{m}\right)$ such that $v_{m}^{n} \stackrel{*}{\rightarrow} u_{n}$ in $W^{1, \infty}$ as $m \rightarrow+\infty$, and

$$
\limsup _{m \rightarrow+\infty} \int_{\Omega_{n}} f\left(\nabla v_{m}^{n}(x)\right) \mathrm{d} x \leq \int_{\Omega_{n}} \mathcal{Z} f\left(\nabla u_{n}(x)\right) \mathrm{d} x .
$$

By compact imbedding theorem for every $n \in \mathbb{N}^{*}$ there exists a subsequence (not relabelled) $v_{m}^{n} \rightarrow u_{n}$ in $L^{1}\left(\Omega_{n} ; \mathbb{R}^{m}\right)$. Set $u_{m, n}=\mathbb{I}_{\Omega_{n}} v_{m}^{n}+\mathbb{I}_{\Omega \backslash \Omega_{n}} u_{n}$. From (6.1), we have

$$
\limsup _{m \rightarrow+\infty} \int_{\Omega} f\left(\nabla u_{m, n}(x)\right) \mathrm{d} x-\int_{\Omega \backslash \Omega_{n}} f\left(\nabla u_{m, n}(x)\right) \mathrm{d} x \leq \int_{\Omega} \mathcal{Z} f\left(\nabla u_{n}(x)\right) \mathrm{d} x .
$$

By $\left(\mathscr{H}_{1}\right)$

We deduce

$$
\left|\int_{\Omega \backslash \Omega_{n}} f\left(\nabla u_{m, n}(x)\right) \mathrm{d} x\right| \leq M_{t}\left|\Omega \backslash \Omega_{n}\right|=\frac{1}{n} M_{t} .
$$

$$
\left\{\begin{array}{c}
\limsup _{n \rightarrow+\infty} \limsup _{m \rightarrow+\infty} \int_{\Omega} f\left(\nabla u_{m, n}(x)\right) \mathrm{d} x \leq \limsup _{n \rightarrow+\infty} \int_{\Omega} \mathcal{Z} f\left(\nabla u_{n}(x)\right) \mathrm{d} x \\
u+W_{0}^{1, \infty}\left(\Omega ; \mathbb{R}^{m}\right) \ni u_{m, n} \rightarrow u_{n} \text { in } L^{1} \text { as } m \rightarrow+\infty .
\end{array}\right.
$$

Since $\nabla u_{n}(x), \nabla u(x) \in \frac{1+t}{2} \bar{C}$ a.e. in $\Omega$ for all $n \geq n_{t}$. Using continuity of $\mathcal{Z} f\left\lfloor_{\text {int } C}\right.$ (see Lem. 2.3), and Lebesgue dominated convergence theorem, we obtain

$$
\left\{\begin{array}{c}
\limsup _{n \rightarrow+\infty} \limsup _{m \rightarrow+\infty} \int_{\Omega} f\left(\nabla u_{m, n}(x)\right) \mathrm{d} x \leq \int_{\Omega} \mathcal{Z} f(\nabla u(x)) \mathrm{d} x \\
u+W_{0}^{1, \infty}\left(\Omega ; \mathbb{R}^{m}\right) \ni u_{m, n} \rightarrow u_{n} \text { in } L^{1} \text { as } m \rightarrow+\infty \\
u_{n} \rightarrow u \text { in } L^{1} \text { as } n \rightarrow+\infty .
\end{array}\right.
$$

Diagonalization arguments give the result.

Lemma 6.2. Assume that $\left(\mathscr{H}_{0}\right)$ and $\left(\mathscr{H}_{1}\right)$ hold. Assume that for every $u \in W^{1, \infty}\left(\Omega ; \mathbb{R}^{m}\right)$ and $t \in[0,1[$ such that $\nabla u(x) \in t \bar{C}$ a.e. in $\Omega$ it holds

Then for every $u \in W^{1, \infty}\left(\Omega ; \mathbb{R}^{m}\right)$

$$
\mathcal{I}(u) \leq \int_{\Omega} \mathcal{Z} f(\nabla u(x)) \mathrm{d} x .
$$

$$
\mathcal{I}(u) \leq \int_{\Omega} \widehat{\mathcal{Z f}}(\nabla u(x)) \mathrm{d} x
$$


Proof. Without loss of generality, assume that

$$
\int_{\Omega} \widehat{\mathcal{Z f}}(\nabla u(x)) \mathrm{d} x<+\infty
$$

with $u \in W^{1, \infty}\left(\Omega ; \mathbb{R}^{m}\right)$. We deduce that $\nabla u(x) \in \bar{C}$ a.e. in $\Omega$ since Lemma 2.2 (ii). Let $n \geq 1$. Set $u_{n}=$ $\left(1-\frac{1}{n}\right) u \in W^{1, \infty}\left(\Omega ; \mathbb{R}^{m}\right)$, then $u_{n} \rightarrow u$ in $L^{1}$. By l.s.p., it holds that $\nabla u_{n}(x) \in\left(1-n^{-1}\right) \bar{C} \subset \operatorname{int} C$ a.e. in $\Omega$. Let $\varepsilon>0$. By Lemma 2.5, there exists $\eta>0$ such that for every $\xi \in \bar{C}$ and $t \in[0,1[$, if $1-t<\eta$ then $\mathcal{Z} f(t \xi) \leq \widehat{\mathcal{Z} f}(\xi)+\varepsilon$. Choose $n(\varepsilon) \in \mathbb{N}^{*}$ such that $1-n^{-1}<\eta$ for all $n \geq n(\varepsilon)$. Thus, for every $n \geq n(\varepsilon)$

$$
\mathcal{I}\left(u_{n}\right) \leq \int_{\Omega} \mathcal{Z} f\left(\nabla u_{n}(x)\right) \mathrm{d} x \leq \varepsilon|\Omega|+\int_{\Omega} \widehat{\mathcal{Z} f}(\nabla u(x)) \mathrm{d} x .
$$

Letting $n \rightarrow+\infty$ and using the $L^{1}$ sequential lower semicontinuity of $\mathcal{I}$, we have

$$
\mathcal{I}(u) \leq \varepsilon|\Omega|+\int_{\Omega} \widehat{\mathcal{Z} f}(\nabla u(x)) \mathrm{d} x .
$$

Since $\varepsilon>0$ is arbitrary, we obtain the desired result.

\subsection{End of the proofs of Theorems 1.2 and 1.4}

6.1.1. Proof of the lower bound

Proposition 6.1. Let $p \in[1,+\infty]$. Assume that $\left(\mathscr{H}_{0}\right)$ holds. Assume that either $\left(\mathscr{H}_{1}\right)$ and $\left(\mathscr{H}_{2}\right)$ or $\left(\mathscr{H}_{3}\right)$ and $\left(\mathscr{H}_{4}\right)$ hold. Then for every $u,\left\{u_{n}\right\}_{n \in \mathbb{N}} \subset W^{1, p}\left(\Omega ; \mathbb{R}^{m}\right)$, if $u_{n} \rightarrow u$ in $W^{1, p}\left(u_{n} \stackrel{*}{\rightarrow} u\right.$ in $W^{1, \infty}$ if $\left.p=+\infty\right)$ then

$$
\liminf _{n \rightarrow+\infty} \int_{\Omega} \mathcal{Q} f\left(\nabla u_{n}(x)\right) \mathrm{d} x \geq \int_{\Omega} \mathcal{Q} f(\nabla u(x)) \mathrm{d} x .
$$

Proof. Let $\left\{u_{n}\right\}_{n \geq 1}, u \in W^{1, p}\left(\Omega ; \mathbb{R}^{m}\right)$ such that $u_{n} \rightarrow u$ in $W^{1, p}$. By either Theorems 1.1 or 1.3 , there is a nondecreasing sequence of quasiconvex functions with linear growth conditions $\left\{f_{k}\right\}_{k \in \mathbb{N}}$ satisfying $\sup _{k \in \mathbb{N}} f_{k}(\xi)=$ $\mathcal{Q} f(\xi)$ for all $\xi \in \mathbb{M}^{m \times d}$. Without loss of generality, assume that

$$
\liminf _{n \rightarrow+\infty} \int_{\Omega} \mathcal{Q} f\left(\nabla u_{n}(x)\right) \mathrm{d} x<+\infty .
$$

Then by monotone convergence theorem together with Acerbi and Fusco [1] (lower semicontinuity results), Theorems [II.4] and [II.1], we obtain

$$
\begin{aligned}
\liminf _{n \rightarrow+\infty} \int_{\Omega} \mathcal{Q} f\left(\nabla u_{n}(x)\right) \mathrm{d} x & =\liminf _{n \rightarrow+\infty} \sup _{k \in \mathbb{N}} \int_{\Omega} f_{k}\left(\nabla u_{n}(x)\right) \mathrm{d} x \\
& \geq \liminf _{n \rightarrow+\infty} \int_{\Omega} f_{k}\left(\nabla u_{n}(x)\right) \mathrm{d} x \geq \int_{\Omega} f_{k}(\nabla u(x)) \mathrm{d} x
\end{aligned}
$$

for all $k \in \mathbb{N}$. The proof follows by applying again the monotone convergence theorem.

Lemma 6.3. Let $u_{0} \in W^{1, \infty}\left(\Omega ; \mathbb{R}^{m}\right)$. Let $I^{\infty}, I_{0}^{\infty}, \mathcal{I}_{0}: L^{1}\left(\Omega ; \mathbb{R}^{m}\right) \rightarrow[0,+\infty]$ be three functionals defined by

$$
\begin{aligned}
& I^{\infty}(u)=\inf \left\{\liminf _{n \rightarrow+\infty} \int_{\Omega} f\left(\nabla u_{n}(x)\right) \mathrm{d} x: W^{1, \infty}\left(\Omega ; \mathbb{R}^{m}\right) \ni u_{n} \stackrel{*}{\rightarrow} u \text { in } W^{1, \infty}\right\}, \\
& I_{0}^{\infty}(u)=\inf \left\{\liminf _{n \rightarrow+\infty} \int_{\Omega} f\left(\nabla u_{n}(x)\right) \mathrm{d} x: u_{0}+W_{0}^{1, \infty}\left(\Omega ; \mathbb{R}^{m}\right) \ni u_{n} \stackrel{*}{*} u \text { in } W^{1, \infty}\right\},
\end{aligned}
$$


and

Then

$$
\mathcal{I}_{0}(u)=\inf \left\{\liminf _{n \rightarrow+\infty} \int_{\Omega} f\left(\nabla u_{n}(x)\right) \mathrm{d} x: u_{0}+W_{0}^{1, \infty}\left(\Omega ; \mathbb{R}^{m}\right) \ni u_{n} \rightarrow u \text { in } L^{1}\right\} .
$$

(i) if $\Omega$ is connected and Lipschitz then $\mathcal{I}=I^{\infty}$;

(ii) if $\Omega$ is Lipschitz then $I_{0}^{\infty}=\mathcal{I}_{0}$.

Proof. (i) By compact imbedding of $W^{1, \infty}\left(\Omega ; \mathbb{R}^{m}\right)$ in $L^{1}\left(\Omega ; \mathbb{R}^{m}\right)$ it holds $\mathcal{I} \leq I^{\infty}$. Using Lemma 4.4.2. in [6] and the fact that $\operatorname{dom} f$ is bounded, we deduce $\mathcal{I}=I^{\infty}$.

(ii) As above $\mathcal{I}_{0} \leq I_{0}^{\infty}$. Using Theorem 4.3.18 in [6], we obtain the equality.

By Lemma 6.3 (i) (resp. (ii)), note that $\mathcal{I}(u)=+\infty\left(\right.$ resp. $\left.\mathcal{I}_{0}(u)=+\infty\right)$ if $u \in L^{1}\left(\Omega ; \mathbb{R}^{m}\right) \backslash W^{1, \infty}\left(\Omega ; \mathbb{R}^{m}\right)$ (resp. $u \in L^{1}\left(\Omega ; \mathbb{R}^{m}\right) \backslash u_{0}+W_{0}^{1, \infty}\left(\Omega ; \mathbb{R}^{m}\right)$ ).

Now, we can finish the proof of the lower bound. By Lemma 6.3 (i), the inequality $\mathcal{Q} f \leq f$ and Proposition 6.1, it follows that for every $u \in W^{1, \infty}\left(\Omega ; \mathbb{R}^{m}\right)$

$$
\begin{aligned}
\mathcal{I}(u)=I^{\infty}(u) & \geq \inf \left\{\liminf _{n \rightarrow+\infty} \int_{\Omega} \mathcal{Q} f\left(\nabla u_{n}\right) \mathrm{d} x: W^{1, \infty}\left(\Omega ; \mathbb{R}^{m}\right) \ni u_{n} \stackrel{*}{\rightarrow} u \text { in } W^{1, \infty}\right\} \\
& \geq \int_{\Omega} \mathcal{Q} f(\nabla u(x)) \mathrm{d} x .
\end{aligned}
$$

The proof is complete.

\subsubsection{Proof of the upper bound}

Using Lemmas 6.1, 6.2 and either Theorems 1.1 or 1.3 , we obtain for every $u \in W^{1, \infty}\left(\Omega ; \mathbb{R}^{m}\right)$

$$
\mathcal{I}(u) \leq \int_{\Omega} \mathcal{Q} f(\nabla u(x)) \mathrm{d} x .
$$

Remark 6.1. Let $u_{0} \in W^{1, \infty}\left(\Omega ; \mathbb{R}^{m}\right)$. If we assume that $\Omega$ is Lipschitz only. Using similar arguments as in the proofs of the lower and the upper bound, and the Lemma 6.3 (ii) we can prove that

$$
\mathcal{I}_{0}(u)=\left\{\begin{array}{cl}
\int_{\Omega} \mathcal{Q} f(\nabla u(x)) \mathrm{d} x & \text { if } u \in u_{0}+W_{0}^{1, \infty}\left(\Omega ; \mathbb{R}^{m}\right) \\
+\infty & \text { if } u \in L^{1}\left(\Omega ; \mathbb{R}^{m}\right) \backslash u_{0}+W_{0}^{1, \infty}\left(\Omega ; \mathbb{R}^{m}\right) .
\end{array}\right.
$$

\section{A. Some Results about $\mathcal{Z} g$}

Let $g: \mathbb{M}^{m \times d} \rightarrow[0,+\infty]$ be a Borel measurable function.

Lemma A.1 (Fonseca [10], Lem. 2.16). For every bounded open set $D \subset \mathbb{R}^{d}$ satisfying $|\partial D|=0$ it holds

$$
\mathcal{Z} g(\xi)=\inf \left\{\frac{1}{|D|} \int_{D} g(\xi+\nabla \phi(x)) \mathrm{d} x: \phi \in W_{0}^{1, \infty}\left(D ; \mathbb{R}^{m}\right)\right\} .
$$

Lemma A.2. If $\mathcal{Z} g$ is finite then $\mathcal{Z} g=\mathcal{Q} g$.

Proof. On one hand, we have $\mathcal{Z} g \geq \mathcal{Q} g$, it follows that $\mathcal{Q}(\mathcal{Z} g) \geq \mathcal{Q} g$, since $\mathcal{Q} g$ is finite and then quasiconvex. On the other hand, $g \geq \mathcal{Z} g \geq \mathcal{Q}(\mathcal{Z} g)$ and then $\mathcal{Q} g \geq \mathcal{Q}(\mathcal{Z} g)$ since $\mathcal{Q}(\mathcal{Z} g)$ is finite and then quasiconvex. Therefore $\mathcal{Q}(\mathcal{Z} g)=\mathcal{Q} g$. We are reduced to prove that $\mathcal{Z} g$ is quasiconvex. Using the same arguments as in the proof of Lemma 2.3, we have $\mathcal{Z} g$ is continuous since it is finite. Let $\xi \in \mathbb{M}^{m \times d}$ and $\phi \in W_{0}^{1, \infty}\left(Y ; \mathbb{R}^{m}\right)$. Without loss of generality we can assume that $\int_{Y} g(\xi+\nabla \phi(x)) \mathrm{d} x<+\infty$. By [7], Theorem 1.8, there exists 
$\left\{\phi_{n}\right\}_{n \geq 1} \in \operatorname{Aff}_{0}\left(Y ; \mathbb{R}^{m}\right)$ such that $\nabla \phi_{n} \rightarrow \nabla \phi$ a.e. in $Y$ and $\sup _{n \geq 1}\left|\nabla \phi_{n}\right|_{\infty} \leq C|\nabla \phi|_{\infty}$ for some $C>0$. Thus by continuity of $\mathcal{Z} g$ we have

$$
\left\{\begin{aligned}
\mathcal{Z} g\left(\xi+\nabla \phi_{n}(x)\right) & \leq \sup _{|\zeta| \leq c} \mathcal{Z} g(\xi+\zeta)<+\infty \text { a.e. in } Y \\
\mathcal{Z} g\left(\xi+\nabla \phi_{n}(x)\right) & \rightarrow \mathcal{Z} g(\xi+\nabla \phi(x)) \text { a.e. in } Y
\end{aligned}\right.
$$

for some $c>0$. Applying Lebesgue dominated convergence theorem we obtain

$$
\lim _{n \rightarrow+\infty} \int_{Y} \mathcal{Z} g\left(\xi+\nabla \phi_{n}(x)\right) \mathrm{d} x=\int_{Y} \mathcal{Z} g(\xi+\nabla \phi(x)) \mathrm{d} x .
$$

Let $\varepsilon>0$. There is $n_{0} \geq 1$ such that for every $n \geq n_{0}$

$$
\int_{Y} \mathcal{Z} g\left(\xi+\nabla \phi_{n}(x)\right) \mathrm{d} x \leq \int_{Y} \mathcal{Z} g(\xi+\nabla \phi(x)) \mathrm{d} x+\varepsilon .
$$

Fix $n \geq n_{0}$. For some finite family $\left\{\xi_{i}^{n}\right\}_{i \in I^{n}} \subset \mathbb{M}^{m \times d}$ and disjoints open bounded sets $\left\{U_{i}^{n}\right\}_{i \in I^{n}}$ with $\left|\partial U_{i}^{n}\right|=0$ and $\left|Y \backslash \cup_{i \in I_{n}} U_{i}^{n}\right|=0$, we can write $\nabla \phi_{n}=\sum_{i \in I^{n}} \xi_{i}^{n} \mathbb{I}_{U_{i}^{n}}$ a.e. in $Y$. By Lemma A.1, we have that for any $i \in I^{n}$ there exists $\varphi_{i}^{n} \in W_{0}^{1, \infty}\left(U_{i}^{n} ; \mathbb{R}^{m}\right)$ such that

$$
\varepsilon+\mathcal{Z} g\left(\xi+\xi_{i}^{n}\right) \geq \frac{1}{\left|U_{i}^{n}\right|} \int_{U_{i}^{n}} g\left(\xi+\xi_{i}^{n}+\nabla \varphi_{i}^{n}(x)\right) \mathrm{d} x .
$$

Set $\varphi_{n}(x)=\varphi_{i}^{n}(x)$ if $x \in U_{i}^{n}$, and $\varphi_{i}^{n}(x)=0$ otherwise. Then $\varphi_{n}+\phi_{n} \in W_{0}^{1, \infty}\left(Y ; \mathbb{R}^{m}\right)$ and

$$
\begin{aligned}
\int_{Y} \mathcal{Z} g(\xi+\nabla \phi) \mathrm{d} x+\varepsilon & \geq \varepsilon+\int_{Y} \mathcal{Z} g\left(\xi+\nabla \phi_{n}\right) \mathrm{d} x \geq \sum_{i \in I^{n}} \int_{U_{i}^{n}} g\left(\xi+\xi_{i}^{n}+\nabla \varphi_{i}^{n}\right) \mathrm{d} x \\
& \geq \int_{Y} g\left(\xi+\nabla \phi_{n}+\nabla \varphi_{n}\right) \mathrm{d} x \geq \mathcal{Z} g(\xi) .
\end{aligned}
$$

Letting $\varepsilon \rightarrow 0$, we obtain that $\mathcal{Z} g$ is quasiconvex and the proof is finished.

\section{REFERENCES}

[1] E. Acerbi and N. Fusco, Semicontinuity problems in the calculus of variations. Arch. Rational Mech. Anal. 86 (1984) $125-145$.

[2] O. Anza Hafsa and J.-P. Mandallena, Relaxation of variational problems in two-dimensional nonlinear elasticity. Ann. Mat. Pura Appl. 186 (2007) 187-198.

[3] O. Anza Hafsa and J.-P. Mandallena, Relaxation theorems in nonlinear elasticity. Ann. Inst. H. Poincaré, Anal. Non Linéaire 25 (2008) 135-148.

[4] J.M. Ball and F. Murat, $W^{1, p}$-quasiconvexity and variational problems for multiple integrals. J. Funct. Anal. 58 (1984) $225-253$.

[5] H. Ben Belgacem, Relaxation of singular functionals defined on Sobolev spaces. ESAIM: COCV 5 (2000) 71-85.

[6] L. Carbone and R. De Arcangelis, Unbounded functionals in the calculus of variations, Representation, relaxation, and homogenization, Monographs and Surveys in Pure and Applied Mathematics 125. Chapman \& Hall/CRC, Boca Raton, FL, USA (2002).

[7] B. Dacorogna, Direct methods in the Calculus of Variations. Springer-Verlag (1989).

[8] B. Dacorogna and P. Marcellini, General existence theorems for Hamilton-Jacobi equations in the scalar and vectorial cases. Acta Math. 178 (1997) 1-37.

[9] B. Dacorogna and P. Marcellini, Implicit partial differential equations, Progress in Nonlinear Differential Equations and their Applications 37. Birkhäuser Boston, Inc., Boston, MA, USA (1999).

[10] I. Fonseca, The lower quasiconvex envelope of the stored energy function for an elastic crystal. J. Math. Pures Appl. 67 (1988) $175-195$. 
[11] D. Kinderlehrer and P. Pedregal, Characterizations of Young measures generated by gradients. Arch. Rational Mech. Anal. 115 (1991) 329-365.

[12] C.B. Morrey, Quasiconvexity and lower semicontinuity of multiple integrals. Pacific J. Math. 2 (1952) 25-53.

[13] S. Müller, A sharp version of Zhang's theorem on truncating sequences of gradients. Trans. Amer. Math. Soc. 351 (1999) 4585-4597.

[14] T.R. Rockafellar and J.-B. Wets, Variational analysis, Grundlehren der Mathematischen Wissenschaften [Fundamental Principles of Mathematical Sciences] 317. Springer-Verlag, Berlin (1998).

[15] M. Wagner, On the lower semicontinuous quasiconvex envelope for unbounded integrands. To appear on ESAIM: COCV (to appear).

[16] M. Wagner, Quasiconvex relaxation of multidimensional control problems. Adv. Math. Sci. Appl. (to appear). 